\title{
Symmetry and Related Properties via the Maximum Principle
}

\author{
B. Gidas ${ }^{1 \star}$, Wei-Ming $\mathrm{Ni}^{2}$, and L. Nirenberg ${ }^{2 \star \star}$ \\ ${ }^{1}$ Rockefeller University and ${ }^{2}$ Courant Institute of Math. Sci., New York University. New York. \\ NY 10012 USA
}

\begin{abstract}
We prove symmetry, and some related properties, of positive solutions of second order elliptic equations. Our methods employ various forms of the maximum principle, and a device of moving parallel planes to a critical position, and then showing that the solution is symmetric about the limiting plane. We treat solutions in bounded domains and in the entire space.
\end{abstract}

\section{Introduction}

1.1 In an elegant paper [8], Serrin considered solutions of second order elliptic equations satisfying over-determined boundary conditions. For equations with spherical symmetry he proved that the domain on which the solution is defined is necessarily a ball and that the solution is spherically symmetric. The proof is based on the maximum principle and on a device (which goes back to Alexandroff; see Chap. 7 in [3]) of moving parallel planes to a critical position and then showing that the solution is symmetric about the limiting plane.

In this paper we will use the same technique to prove symmetry of positive solutions of elliptic equations vanishing on the boundary - as well as related results (including some extensions to parabolic equations). Some of the equations we treat are related to physics and our techniques should be applicable to other physical situations. We study solutions in bounded domains and in the entire space. The simplest example in a bounded domain is

Theorem 1. In the ball $\Omega:|x|<R$ in $\mathbb{R}^{n}$, let $u>0$ be a positive solution in $C^{2}(\bar{\Omega})$ of

$$
\Delta u+f(u)=0 \text { with } u=0 \text { on }|x|=R .
$$

Here $f$ is of class $C^{1}$. Then $u$ is radially symmetric and

$$
\frac{\partial u}{\partial r}<0, \text { for } 0<r<R .
$$

* Supported in part by the National Science Foundation, grant no. PHY-78-08066

$\star \star$ Partially supported by the U.S. Army Research Office, grant no. DAA 29-78-G-0127 
The point of interest is that the result holds no matter what $f$ is. We should note that $f(u) \geqq 0$ for all $u$, implies that any nontrivial solution is automatically positive in $\Omega$.

Theorem 1 is a special case of Theorem $1^{\prime}$ in Sect. 2. Another simple special result is :

Theorem 2. Let $u>0$ be a $C^{2}$ solution of (1.1) in a ring-shaped domain

$$
R^{\prime}<|x| \leqq R .
$$

Then

$$
\frac{\partial u}{\partial r}<0 \quad \text { for } \quad \frac{R^{\prime}+R}{2} \leqq|x|<R .
$$

This implies that $u$ can have no critical point in this larger half of the ring. Note that no condition is imposed at the boundary $|x|=R^{\prime}$.

A third simple result (see Theorem $3^{\prime}$ in Sect. 3 for a more general form) is :

Theorem 3. Let $u(x)$ be a $C^{2}$ solution of the ordinary differential equation

$$
\ddot{u}+b(x) \dot{u}+f(u)=0 \quad \text { on } \quad 0<x<1,
$$

with $u$ continuous on $0<x \leqq 1$ and $u(x)>u(1)$ for $0<x<1$. Here $f \in C^{1}$, and $b(x)$ is continuous in $0<x<1$. If $b(x) \geqq 0$ everywhere then

$$
\dot{u}<0 \quad \text { on } \quad \frac{1}{2}<x<1 \text {. }
$$

Furthermore if $\dot{u}\left(\frac{1}{2}\right)=0$ then $u$ is symmetric about $\frac{1}{2}$ and $b(x)$ is necessarily identically zero.

As an example: $u=1-\cos 2 \pi x$ is a solution of

$$
\ddot{u}+4 \pi^{2}(u-1)=0, \quad 0 \leqq x \leqq 1
$$

satisfying all the conditions of the theorem.

1.2 Our interest in these questions grew out of a study of positive solutions in $\mathbb{R}^{n}$, $n>2(n=4$ in particular) of the equation

$$
\Delta u+u^{\frac{n+2}{n-2}}=0 \text {. }
$$

This is the Euler equation for the function ("action")

$$
A(u)=\int_{R^{n}}\left(\frac{1}{2}|\operatorname{grad} u|^{2}-\frac{u^{\beta}}{\beta}\right) d x, \quad \beta=\frac{2 n}{n-2} .
$$

Equation (1.5) and the associated action are conformally invariant in the sense that if $u$ is a solution, then after a conformal mapping $x \rightarrow y$ the function

$$
v(y)=u(x) J^{\frac{2-n}{2 n}}(x),
$$

where $J$ is the Jacobian, is also a solution. For $n=4$, Eq. (1.5) as well as the corresponding, but simpler, equation $\Delta u=u^{\frac{n+2}{n-2}}$ studied in [5], and the linear 
equation $u=0$, give rise to bona-fide solutions of the classical Euclidean YangMills equations via 't Hooft's Ansatz [1,9]. Equation (1.5) has the explicit solutions (replace $x$ by $i x$ in the solutions of page 250 of [5])

$$
u(x)=\frac{\left[n(n-2) \lambda^{2}\right]^{\frac{n-2}{4}}}{\left(\lambda^{2}+\left|x-x_{0}\right|^{2}\right)^{n / 2-1}}
$$

for $\lambda>0, x_{0} \in R^{n}$. These solutions yield the well-known one-instanton solutions in a regular gauge of the Yang-Mills equations. We used the same methods as in the proofs of the preceding results to show that these are the only positive solutions in $R^{n}$ with reasonable behaviour at infinity, namely $u=0\left(|x|^{2-n}\right)$. This behaviour follows from the finiteness of the action - as was proved by K. Uhlenbeck (private communication). This uniqueness result together with the well known properties (see [5]) of $\Delta u=u^{(n+2) /(n-2)}$ and of $\Delta u=0([4])$ show that any finite action solution of the full Yang-Mills equations given by 't Hooft's Ansatz is self-dual.

Subsequently it was brought to our attention by R. Schoen that our uniqueness result is equivalent to the following geometric result due to Obata [6]: A Riemannian metric on $S^{n}$ which is conformal to the usual one and having the same constant scalar curvature, is in fact the pullback of the usual one under a conformal map of $S^{n}$ to itself.

As a demonstration of the use of our methods in the full space we present a related result for equations which are rotationally, but not necessarily conformally, invariant. For convenience we suppose $n>2$.

Theorem 4. Let $v>0$ be a $C^{2}$ solution of an elliptic equation

$$
F\left(v,|\operatorname{grad} v|^{2}, \sum v_{j} v_{k} v_{j k}, \operatorname{tr} A, \operatorname{tr} A^{2}, \ldots, \operatorname{tr} A^{n}\right)=0 \quad \text { in } \quad R^{n}
$$

where $A=$ the Hessian matrix $\left\{v_{i j}\right\}$, here $F \in C^{1}$ for $v>0$ and all values of the other arguments.

Assume that near infinity, $v$ and its first derivatives admit the asymptotic expansion (using summation convention):

$$
\begin{aligned}
v & =\frac{1}{|x|^{m}}\left(a_{0}+\frac{a_{j} x_{j}}{|x|^{2}}+\frac{a_{j k} x_{j} x_{k}}{|x|^{4}}+o\left(\frac{1}{|x|^{2}}\right)\right) \\
v_{x_{2}} & =-\frac{m}{|x|^{m+2}} x_{i}\left(a_{0}+\frac{a_{j} x_{j}}{|x|^{2}}\right)+\frac{a_{i}}{|x|^{m+2}}-\frac{2 x_{i}}{|x|^{m+4}} a_{j} x_{j}+O\left(\frac{1}{|x|^{m+3}}\right)
\end{aligned}
$$

for some $m, a_{0}>0$. Then $v$ is rotationally symmetric about some point and $v_{r}<0$ for $r>0$ where $r$ is the radial coordinate about that point.

Here, to say that an equation $G\left(x, v, v_{i}, v_{j k}\right)=0$ is elliptic means $G_{v_{j k}}$ is a positive definite matrix for all values of the arguments.

It is natural to ask under what conditions one can assert that expansions of the form (1.9) hold. Here is an example:

Proposition 1. Let $v \in C^{2+\mu}, 0<\mu<1$, be a positive solution of

$$
\Delta v+f(v)=0
$$


with $v(x)=O\left(|x|^{2-n}\right)$ as $x \rightarrow \infty$. Assume that for some $k \geqq \frac{n+2}{n-2}, g(v)=f(v) v^{-k}$ is Hölder continuous on $0 \leqq v \leqq v_{0}$ where $v_{0}$ is the maximum of our solution. Then expansions (1.9), with $m=n-2$, hold for $|x|$ large.

Example. The function

$$
v(x)=\left(1+|x|^{4}\right)^{-(n-2) / 4}
$$

satisfies $(1.8)^{\prime}$ with

$$
f(v)=\left(n^{2}-4\right) v^{\frac{n+6}{n-2}}\left(v^{\frac{4}{2-n}}-1\right)^{1 / 2} .
$$

This satisfies the conditions of the proposition with $k=\frac{n+4}{n-2}$.

1.3 Our results are based on a well-known form of the maximum principle [7] for a $C^{2}$ solution $u \leqq 0$ of the differential inequality (we use summation convention)

$$
L u \equiv a_{i j}(x) u_{x_{i} x_{j}}+b_{i}(x) u_{x_{1}}+c(x) u \geqq 0
$$

in a domain $\Omega$ (open connected subset of $R^{n}$ ), and the corresponding Hopf boundary lemma [7]. Here $L$ is a uniformly elliptic operator, i.e., for some constant $c_{0}>0$

$$
a_{i j} \xi_{i} \xi_{j} \geqq c_{0}|\xi|^{2}
$$

and the coefficients of $L$ are uniformly bounded in absolute value.

Maximum Principle. If $u$ vanishes at some point in $\Omega$ then $u \equiv 0$.

We use the Hopf lemma in the form:

Lemma H. Suppose there is a ball $B$ in $\Omega$ with a point $P \in \partial \Omega$ on its boundary and suppose $u$ is continuous in $\Omega \cup P$ and $u(P)=0$. Then if $u \neq 0$ in $B$ we have for an outward directional derivative at $P$,

$$
\frac{\partial u}{\partial v}(P)>0,
$$

in the sense that if $Q$ approaches $P$ in $B$ along a radius then

$$
\lim _{Q \rightarrow P} \frac{u(P)-u(Q)}{|P-Q|}>0 \text {. }
$$

This is well known in case $c(x) \leqq 0$ (see Theorem 7, p. 65 of [7]) but, as already observed by Serrin in [8] p. 310, the more general result follows by the same argument used to prove the maximum principle in the form above. For the convenience of the reader we include the derivation, using the well known result for case $c \equiv 0$.

Proof. With

$$
v=e^{-\alpha x_{1}} u, \quad \alpha>0
$$


one obtains

$$
0 \leqq L u=e^{\alpha x_{1}} L^{\prime} v+v L\left(e^{\alpha x_{1}}\right)
$$

where $L^{\prime}$ is an elliptic operator containing no zero-order term. Thus

$$
0 \leqq L^{\prime} v+v\left(a_{11} \alpha^{2}+b_{1} \alpha+c\right)=L^{\prime} v+c^{\prime} v .
$$

For $\alpha$ sufficiently large, $c^{\prime} \geqq 0$. Hence, since $v \leqq 0$,

$$
L^{\prime} v \geqq 0 \quad \text { in } \Omega .
$$

As $v(P)=0$ we have by the usual form of the Hopf lemma,

$$
\frac{\partial v}{\partial v}(P)>0,
$$

and the desired result follows since $u_{v}(P)=e^{\alpha x_{1}} v_{v}(P)$.

We shall also use the following consequence of the maximum principle.

Corollary. Suppose in (1.10) that for some positive constants $m, b, c_{1}$

$$
a_{11} \geqq m>0, \quad b_{1} \geqq-b, \quad c \leqq c_{1} .
$$

Assume that $\bar{\Omega}$ lies in a narrow region

$$
a-\varepsilon<x_{1}<a,
$$

and $u$ is a solution of the inequality (1.10) in $\Omega$ with $u \leqq 0$ on $\partial \Omega$. Then $u \leqq 0$ in $\Omega$ provided

$$
c_{1} \exp (2 b \varepsilon / m) \leqq c_{1}+2 b^{2} / m \text {. }
$$

The proof makes use of arguments in [2] pages 330-331; for convenience we present it here. See also [7] pages 73-74.

Proof. For $\alpha=2 b / m$ the function

$$
g=e^{\alpha a}-e^{\alpha x_{1}}
$$

is positive in $\bar{\Omega}$ and satisfies

$$
\begin{aligned}
-L g & =\left(a_{11} \alpha^{2}+b_{1} \alpha\right) e^{\alpha x_{1}}-c\left(e^{\alpha a}-e^{\alpha x_{1}}\right) \\
& \geqq\left(m \alpha^{2}-b \alpha\right) e^{\alpha x_{1}}-c_{1}\left(e^{\alpha a}-e^{\alpha x_{1}}\right) .
\end{aligned}
$$

Thus

$$
\begin{aligned}
-e^{-\alpha x_{1}} L g & \geqq m \alpha^{2}-b \alpha+c_{1}-c_{1} e^{\alpha \varepsilon} \\
& =\frac{2 b^{2}}{m}+c_{1}-c_{1} e^{\alpha \varepsilon} \quad(\text { since } \alpha=2 b / m), \\
& \geqq 0 \quad \text { by our hypothesis. }
\end{aligned}
$$

Consequently the function

$$
v=\frac{u}{g}
$$


satisfies

$$
L^{\prime} v+v \frac{L g}{g} \geqq 0 \quad \text { in } \quad \Omega
$$

where $L^{\prime}$ is an elliptic operator with no zero-order term. Since $L g / g \leqq 0$ and $v \leqq 0$ on $\partial \Omega$, the usual form of the maximum principle yields $v \leqq 0$, and hence $u \leqq 0$ in $\Omega$.

In Sect. 3 we will also make use of an extension of the Hopf boundary lemma, at a corner, due to Serrin (Lemma 2 in [8]). Since it may be of further interest we present a slightly more general form (requiring however a bit more smoothness of the coefficients):

Lemma $\mathbf{S}$. Let $\Omega$ be a domain in $\mathbb{R}^{n}$ with the origin $O$ on its boundary. Assume that near $O$ the boundary consists of two transversally intersecting $C^{2}$ hypersurfaces $Q=0$ and $\sigma=0$. Suppose $\varrho, \sigma<0$ in $\Omega$. Let $w$ be a function in $C^{2}(\bar{\Omega})$, with $w<0$ in $\Omega$, $w(0)=0$, satisfying the differential inequality (1.10) in $\Omega$ with uniformly bounded coefficients satisfying (1.11). Assume

$$
a_{i j} \varrho_{x_{i}} \sigma_{x_{j}} \geqq 0 \text { at } 0 \text {. }
$$

If this is zero assume furthermore that $a_{i j} \in C^{2}$ in $\bar{\Omega}$ near $O$, and that

$$
D\left(a_{i j} Q_{x_{i}} \sigma_{x_{j}}\right)=0 \text { at } O \text {, }
$$

for any first order derivative $D$ at $O$ tangent to the submanifold $\{\varrho=0\} \cap\{\sigma=0\}$. Then, for any direction s at $O$ which enters $\Omega$ transversally to each hypersurface,

$$
\begin{aligned}
& \frac{\partial w}{\partial s}<0 \text { at } O \text { in case of strict inequality in (1.12), } \\
& \frac{\partial w}{\partial s}<0 \text { or } \frac{\partial^{2} w}{\partial s^{2}}<0 \text { at } O \text { in case of equality in (1.12) }
\end{aligned}
$$

Note that conditions (1.12) and $\left(1.12^{\prime}\right)$ are invariant under change of coordinates, and of the choices of the particular functions $\varrho$ and $\sigma$ representing the bounding hypersurfaces. The proof will be presented in the Appendix together with a rough extension in case (1.12) is weakened (see Lemmas A.1 and A.2).

1.4 In addition to the maximum principle and Lemmas $H$ and $S$ we use the procedure of moving up planes perpendicular to a fixed direction as in [8], and we shall now describe it geometrically.

In the following $\Omega$ is a bounded domain in $\mathbb{R}^{n}$ with smooth boundary. (Some domains with corners will be discussed in $\S 3$.)

Let $\gamma$ be a unit vector in $\mathbb{R}^{n}$ and let $T_{\lambda}$ be the hyperplane $\gamma \cdot x=\lambda$. For $\hat{\lambda}=\tilde{\lambda}$ large, $T$ is disjoint from $\bar{\Omega}$. Let the plane move continuously toward $\Omega$, preserving the same normal, i.e., decrease $\lambda$, until it begins to intersect $\bar{\Omega}$. From that moment on, at every stage the plane $T_{\lambda}$ will cut off from $\Omega$ an open cap $\Sigma(\lambda)$, the part of $\Omega$ on the same side of $T_{\lambda}$ as $T_{\hat{\lambda}}$. Let $\Sigma^{\prime}(\lambda)$ denote the reflection of $\Sigma(\lambda)$ in the plane $T_{\lambda}$. At the beginning, $\Sigma^{\prime}(\lambda)$ will be in $\Omega$ and as $\lambda$ decreases, the reflected cap $\Sigma^{\prime}(\lambda)$ will remain in $\Omega$, at least until one of the following occurs:

(i) $\Sigma^{\prime}(\lambda)$ becomes internally tangent to $\partial \Omega$ at some point $P$ not on $T_{\lambda}$ or 
(ii) $T_{\lambda}$ reaches a position where it is orthogonal to the boundary of $\Omega$ at some point $Q$.

We denote by $T_{\lambda_{1}}: \gamma \cdot x=\lambda_{1}$ the plane $T_{\lambda}$ when it first reaches one of these positions and we call $\Sigma\left(\lambda_{1}\right)=\Sigma_{\gamma}$ the maximal cap associated with $\gamma$. Note that its reflection $\Sigma_{\gamma}^{\prime}$ in $T_{\lambda_{1}}$ lies in $\Omega$.

One of our main results will be that if $u>0$ is a solution of an elliptic equation in $\Omega$ satisfying certain conditions, with $u=0$ on $\partial \Omega$, then $\gamma \cdot \operatorname{grad} u<0$ in $\Sigma_{\gamma}$.

It may be that if we decrease $\lambda$ below $\lambda_{1}$ the reflection $\Sigma^{\prime}(\lambda)$ of $\Sigma(\lambda)$ in the plane $T_{\lambda}$ continuous to belong to $\Omega$ as in the following example:

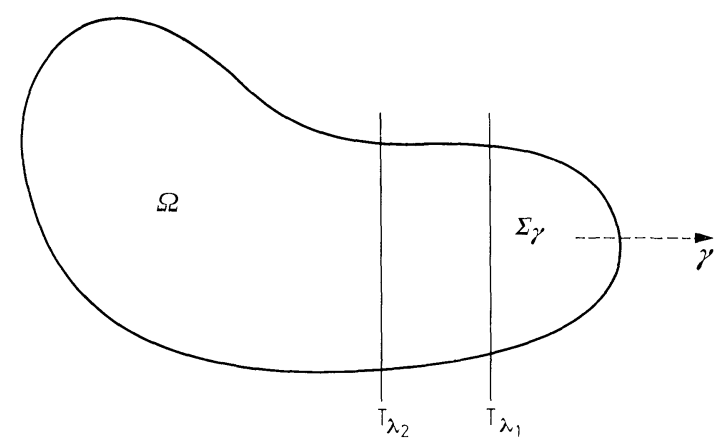

Fig. 1

In that case $\Sigma^{\prime}(\lambda)$ will remain in $\Omega$ for $\lambda$ in a maximal interval

$$
\lambda_{2} \leqq \lambda<\infty, \quad \lambda_{2} \leqq \lambda_{1} .
$$

We will call the cap $\Sigma\left(\lambda_{2}\right)$ the optimal cap corresponding to the direction $\gamma$. Clearly either $\Sigma^{\prime}\left(\lambda_{2}\right)$ is internally tangent to $\partial \Omega$ at some point not on $T_{\lambda_{2}}$, or $T_{\lambda_{2}}$ is orthogonal to $\hat{\partial} \Omega$ at some point.

A word on notation: For a set $S$ in $\partial \Omega$, by a neighbourhood of $S$ in $\Omega$ we mean $\Omega \cap$ (an open neighbourhood of $S$ in $R^{n}$ ).

Section 2 contains the main results for general second order elliptic equations including the proofs of Theorem 1-3. Some extensions are given in 3 and straightforward extensions to parabolic equations are briefly described in Sect. 5. Section 4 is concerned with the proofs of Theorem 4 and Proposition 1 . This may be read independently of the other sections. The Appendix contains the proof of Lemma S.

\section{Principal Results and Proofs}

2.1. Theorems 1 and 2 follow from a single result which, for simplicity, we first present in a special form. The more general form is presented in Theorem 2.1'.

Consider a solution $u \in C^{2}(\Omega)$ of

$$
\Delta u+b_{1}(x) u_{x_{1}}+f(u)=0 \quad \text { in } \Omega
$$


with $b_{1} \in C(\bar{\Omega})$ and $f \in C^{1}$. Here $\Omega$ is bounded with smooth boundary $\partial \Omega$; for $x \in \partial \Omega, v(x)$ is the exterior unit normal.

In our construction of caps in the introduction, let $\gamma$ be the unit vector $(1,0, \ldots, 0)$ and assume $\max _{x \in \Omega} x_{1}=\lambda_{0}$. Let $\Sigma=\Sigma_{\gamma}=\Sigma\left(\lambda_{1}\right)$ be the corresponding maximal cap; the corresponding plane $T_{\lambda_{1}}$ containing part of its boundary is

$$
x_{1}=\lambda_{1}<\lambda_{0} .
$$

The reflection of $\Sigma$ in the plane $T_{\lambda_{1}}$ is called $\Sigma^{\prime}$.

Concerning the solution $u$ we now require

(a) $u>0$ in $\Omega, u \in C^{2}\left(\bar{\Omega} \cap\left\{x_{1}>i_{1}\right\}\right)$, and $u=0$ on $\partial \Omega \cap\left\{x_{1}>\lambda_{1}\right\}$.

Note that no condition on $u$ is required on the rest of the boundary.

For any $x$ in $\Omega$ we use $x^{\lambda}$ to denote its reflection in the plane $T_{\lambda}: x_{1}=\lambda$.

Theorem 2.1. Let $u$ be as above, satisfying condition (a) and assume

$$
b_{1}(x) \geqq 0 \quad \text { in } \quad \Sigma \cup \Sigma^{\prime} .
$$

For any $\lambda$ in $\lambda_{1}<\lambda<\lambda_{0}$ we have

$$
u_{x_{1}}(x)<0 \text { and } u(x)<u\left(x^{\lambda}\right) \text { for } x \in \Sigma(\lambda) .
$$

Thus $u_{x_{1}}<0$ in $\Sigma$. Furthermore if $u_{x_{1}}=0$ at some point in $\Omega$ on the plane $T_{\lambda_{1}}$ then necessarily $u$ is symmetric in the plane $T_{\lambda_{1}}$,

$$
\Omega=\Sigma \cup \Sigma^{\prime} \cup\left(T_{\lambda_{1}} \cap \Omega\right),
$$

and $b_{1}(x) \equiv 0$.

Theorems 1-3 are immediate consequences.

Proof of Theorems 1. Applying Theorem 2.1 we see that

$$
u_{x_{1}}<0 \text { if } x_{1}>0
$$

- for any choice of our $x_{1}$ axis. It follows that $u_{x_{1}}>0$ if $x_{1}<0$. Hence $u_{x_{1}}=0$ on $x_{1}=0$. By the last assertion of Theorem 2.1 we infer that $u$ is symmetric in $x_{1}$. Since the direction of the $x_{1}$ axis is arbitrary it follows that $u$ is radially symmetric and $u_{r}<0$ for $0<r<R$.

Proof of Theorem 2. We may again choose any direction $\gamma$ as positive $x_{1}$ axis. It follows from Theorem 2.1 that in the corresponding maximal cap $\Sigma_{\gamma}, \gamma \cdot \operatorname{grad} u<0$. The union of these maximal caps is the region $\left(R^{\prime}+R\right) / 2<|x|<R$.

Suppose for some point $y$ with $|y|=\left(R^{\prime}+R\right) / 2, u_{r}(y)=0$. Then with $\gamma=y /|y|$ we conclude from the last assertion of Theorem 2.1 that $\Omega=\Sigma_{\gamma} \cup \Sigma_{\gamma}^{\prime}$ which is impossible.

The proof also shows that for $|x|>\left(R^{\prime}+R\right) / 2, v \cdot \operatorname{grad} u(x)<0$ for any vector $v$ making an angle less than $(\pi / 2-\theta)$ with the vector $x$ (see Fig. 2).

Proof of Theorem 3. There are sequences $\varepsilon_{n}, \delta_{n} \rightarrow 0$ such that $u(x)>u\left(1-\varepsilon_{n}\right)$ on the interval $\delta_{n}<x<1-\varepsilon_{n}$. Applying Theorem 2.1 to $u(x)-u\left(1-\varepsilon_{n}\right)$ on that interval and then letting $n \rightarrow \infty$ we easily obtain the result. 


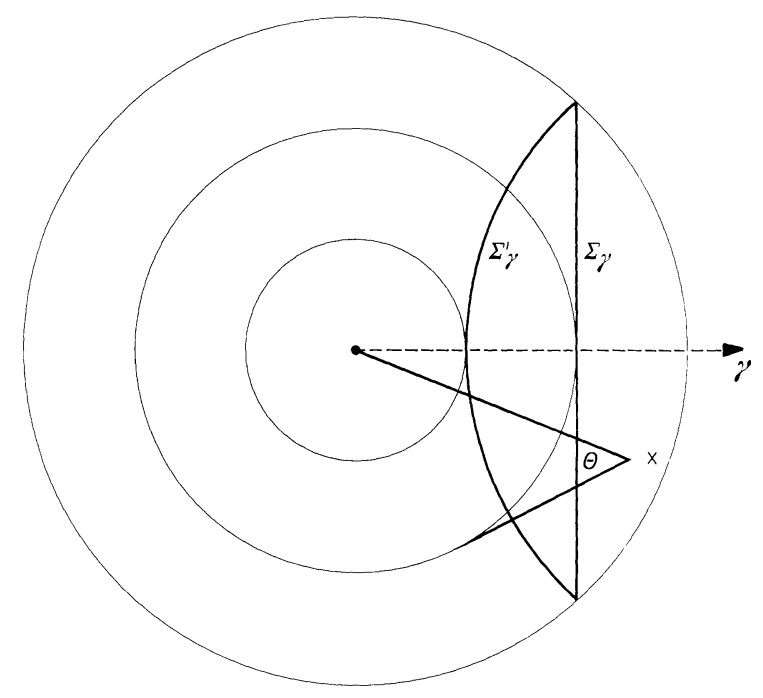

Fig. 2

2.2. For convenience we write $u_{x_{i}}=u_{i}$ etc. Before proving Theorem 2.1 we first apply the maximum principle and Lemma $H$ to derive two preliminary results.

Lemma 2.1. Let $x_{0} \in \partial \Omega$ with $v_{1}\left(x_{0}\right)>0$. For some $\varepsilon>0$ assume $u$ is a $C^{2}$ function in $\bar{\Omega}_{\varepsilon}$ where $\Omega_{\varepsilon}=\Omega \cap\left\{\left|x-x_{0}\right|<\varepsilon\right\}, u>0$ in $\Omega$ and $u=0$ on $\partial \Omega \cap\left\{\left|x-x_{0}\right|<\varepsilon\right\}$. Then $\exists \delta>0$ such that in $\Omega \cap\left\{\left|x-x_{0}\right|<\delta\right\}, u_{x_{1}}<0$.

Proof. Since $u>0$ in $\Omega$, necessarily, $u_{v} \leqq 0$, on $\partial \Omega \cap\left\{\left|x-x_{0}\right|<\varepsilon\right\}=S$, and hence $u_{x_{1}} \leqq 0$ on $S$, for $v_{1}>0$ everywhere there, which we may assume, by decreasing $\varepsilon$ if necessary.

If the lemma were false there would be a sequence of points $x^{j} \rightarrow x_{0}$, with $u_{1}\left(x^{j}\right) \geqq 0$. For $j$ large the interval in the $x_{1}$ direction going from $x^{j}$ to $\partial \Omega$ hits $S$ at a point where $u_{1} \leqq 0$. Consequently, by the theorem of the mean,

$u_{1}\left(x_{0}\right)=0$ and $u_{11}\left(x_{0}\right)=0$.

Suppose $f(0) \geqq 0$. Then in $\Omega_{\varepsilon}, u$ satisfies

$$
\Delta u+b_{1} u_{x_{1}}+f(u)-f(0) \leqq 0
$$

or, by the theorem of the mean, for some function $c_{1}(x)$,

$$
\Delta u+b_{1} u_{1}+c_{1}(x) u \leqq 0 .
$$

Applying Lemma $H$ to the function $-u$ we find

$$
u_{v}\left(x_{0}\right)<0 \text {, and so } u_{1}\left(x_{0}\right)<0
$$

- a contradiction. So suppose $f(0)<0$. From $(2.1)$ we see that at $x_{0}$, $\operatorname{grad} u=0$ and $\Delta u=-f(0)$. But then it follows that

$$
u_{x_{l} x_{j}}=-f(0) v_{i} v_{j} \text { at } x_{0} .
$$


In particular $u_{11}\left(x_{0}\right)>0-$ again a contradiction. The lemma is proved.

Lemma 2.2. Assume that for some $\lambda$ in $\lambda_{1} \leqq \lambda<\lambda_{0}$ we have

$$
u_{1}(x) \leqq 0 \text { and } u(x) \leqq u\left(x^{\lambda}\right) \text { but } u(x) \neq u\left(x^{\lambda}\right) \text { in } \Sigma(\lambda) .
$$

Then $u(x)<u\left(x^{\lambda}\right)$ in $\Sigma(\lambda)$ and $u_{1}(x)<0$ on $\Omega \cap T_{\lambda}$.

Proof. In $\Sigma^{\prime}(\lambda)=$ the reflection of $\Sigma(\lambda)$ in the plane $T_{\lambda}$, consider

$$
v(x)=u\left(x^{\lambda}\right)
$$

note $x^{\lambda} \in \Sigma(\lambda)$. In $\Sigma^{\prime}(\lambda) v$ satisfies the equation

$$
\Delta v-b_{1}\left(x^{\lambda}\right) v_{1}+f(v)=0
$$

and $v_{1} \geqq 0$. If we subtract $(2.1)$ we find

$$
\begin{aligned}
\Delta(v-u)+b_{1}(x)(v-u)_{1}+f(v)-f(u) & =\left(b_{1}\left(x^{\lambda}\right)+b_{1}(x)\right) v_{1} \\
& \geqq 0
\end{aligned}
$$

in $\Sigma^{\prime}(\lambda)$, since $v_{1} \geqq 0$ and $b_{1} \geqq 0$. Using the theorem of the mean in integral form we see that in $\Sigma^{\prime}(\lambda)$

$$
w(x) \equiv v(x)-u(x) \leqq 0, \quad w \neq 0,
$$

and

$$
\Delta w(x)+b_{1}(x) w_{1}+c(x) w \geqq 0
$$

for some function $c(x)$. Since $w=0$ on $T_{\lambda} \cap \Omega$ it follows from the maximum principle and Lemma $H$ that $w<0$ in $\Sigma^{\prime}(\lambda)$ and $w_{1}>0$ on $T_{\lambda}$. But on $T_{\lambda}, w_{1}=v_{1}-u_{1}=-2 u_{1}$, and the lemma is proved.

Proof of Theorem 2.1. It follows from Lemma 2.1 that for $\lambda$ close to $\lambda_{0}, \lambda<\lambda_{0},(2.2)$ holds. Decrease $\lambda$ until a critical value $\mu \geqq \lambda_{1}$ is reached, beyond which it no longer holds. Then (2.2) holds for $\lambda>\mu$, while for $\lambda=\mu$ we have by continuity,

$$
u_{1}(x)<0 \text { and } u(x) \leqq u\left(x^{\mu}\right) \text { for } \quad x \in \Sigma(\mu) .
$$

We will show that $\mu=\lambda_{1}$. Suppose $\mu>\lambda_{1}$. For any point $x_{0} \in \partial \Sigma(\mu) \backslash T_{\mu}$ we have $x_{0}^{\mu} \in \Omega$. Since $0=u\left(x_{0}\right)<u\left(x_{0}^{\mu}\right)$ we see that $u(x) \neq u\left(x^{\mu}\right)$ in $\Sigma(\mu)$. We may therefore apply Lemma 2.2 and conclude that

$$
u(x)<u\left(x^{\mu}\right) \text { in } \Sigma(\mu) \text { and } u_{1}<0 \text { on } \Omega \cap T_{\mu} .
$$

Thus (2.2) holds for $\lambda=\mu$. Since $u_{1}<0$ on $\Omega \cap T_{\mu}$ we see with the aid of Lemma 2.1 that for some $\varepsilon>0$

$$
u_{1}<0 \text { in } \Omega \cap\left\{x_{1}>\mu-\varepsilon\right\} .
$$

From our definition of $\mu$ we must then have the following situation. For $j=1,2, \ldots$ there is a sequence $\lambda^{j}$,

$$
\lambda_{1}<\lambda^{j}>\mu
$$

and a point $x_{j}$ in $\Sigma\left(\lambda^{j}\right)$ such that

$$
u\left(x_{j}\right) \geqq u\left(x_{j}^{\lambda j}\right) .
$$


A subsequence which we still call $x_{j}$ will converge to some point $x$ in $\overline{\Sigma(\mu)}$; then $x_{j}^{\lambda^{\jmath}} \rightarrow x^{\mu}$ and $u(x) \geqq u\left(x^{\mu}\right)$. Since (2.2) holds for $\lambda=\mu$ we must have $x \in \partial \Sigma_{\mu}$. If $x$ is not on the plane $T_{\mu}$ then $x^{\mu}$ lies in $\Omega$ and consequently

$$
0=u(x)<u\left(x^{\mu}\right)
$$

which is impossible. Therefore $x \in T_{\mu}$ and $x^{\mu}=x$. On the other hand, for $j$ sufficiently large, the straight segment joining $x_{j}$ to $x_{j}^{\hat{\lambda}^{\prime}}$ belongs to $\Omega$ and by the theorem of the mean it contains a point $y_{j}$ such that

$$
u_{1}\left(y_{j}\right) \geqq 0 .
$$

Since $y_{j} \rightarrow x$ we obtain a contradiction to (2.5). Thus we have proved that $\mu=\lambda_{1}$ and that (2.2) holds for $\lambda>\lambda_{1}$. By continuity, $u_{1}(x) \leqq 0$ and $u(x) \leqq u\left(x^{\lambda_{1}}\right)$ in $\Sigma$.

To complete the proof of the theorem suppose $u_{1}=0$ at some point in $\Omega$ on $T_{\lambda_{1}}$. By Lemma 2.2 we infer that

$$
u(x) \equiv u\left(x^{\wedge 1}\right) \quad \text { in } \quad \Sigma .
$$

Since $u(x)=0$ if $x \in \partial \Omega$ and $x_{1} \geqq \lambda_{1}$ it follows that $u\left(x^{\lambda_{1}}\right)=0$ at the reflected point and thus (2.3) holds. Finally, suppose $b_{1}>0$ at some point $x \in \Omega$ (we may take $x \notin T_{\lambda_{1}}$ ). Then from the Eq. (2.1) and the (now proved) symmetry of the solution in the plane $T_{\lambda_{1}}$ we see that

$$
b_{1}(x) u_{1}(x)=b_{1}\left(x^{\lambda_{1}}\right) u_{1}\left(x^{\lambda_{1}}\right) .
$$

If $x \in \Sigma$, the left-hand side is negative while the right-hand side is nonnegative impossible; similarly if $x \in \Sigma^{\prime}$. Q.e.d.

Theorem 2.1 admits various generalizations and applications.

2.3. Remark 1. (This was pointed out to us by Spruck.) In some equations of interest, of the form (1.1), the function $f(u)$ is not in $C^{1}$. For example, in a certain plasma problem, $f(u)=(u-1)^{+}=$the positive part of $(u-1)$. In case $f$ is monotone increasing the result of Theorem 1 still holds i.e. we have, more generally:

The results of Theorems 2.1 and 1, and of Lemmas 2.1, 2.2 hold if $f(u)=f_{1}(u)$ $+f_{2}(u)$ where $f_{1} \in C^{1}$ and $f_{2}$ is monotone increasing. In particular the results hold if $f$ is locally Lipschitz continuous.

The proofs are the same as before. We have only to verify that Lemmas 2.1, 2.2 continue to hold; it was only there that the $C^{1}$ hypothesis on $f$ was used. In the proof of Lemma 2.1 the $C^{1}$ hypothesis was used in the argument assuming $f(0) \geqq 0$. In that case in place of $(\widehat{2.1})$ we have

$$
\Delta u+b_{1} u_{1}+f_{1}(u)-f_{1}(0) \leqq f_{2}(0)-f_{2}(u) \leqq 0
$$

since $f_{2}$ is increasing, and the argument proceeds as before. Similarly in the proof of Lemma 2.2 we have from (2.4)

$$
\begin{aligned}
\Delta(v-u)+b_{1}(x)(v-u)_{1}+f_{1}(v)-f_{1}(u) & \geqq f_{2}(u)-f_{2}(v) \\
& \geqq 0
\end{aligned}
$$


and the proof proceeds as before. In fact if $f$ is monotone increasing (i.e., we take $f_{1}=0$ ) we need only the usual form of the Hopf boundary lemma.

It is natural to ask whether Theorems 1 and 2.1 hold if the condition $u>0$ in $\Omega$ is replaced by the condition: $u \geqq 0, u \neq 0$ in $\Omega$. This is not the case in general as we see from the example after Theorem 3 . If however $f(0) \geqq 0$ in both theorems, then $u \geqq 0, u \neq 0$ in $\Omega$ implies $u>0$ in $\Omega$. For, with $f=f_{1}+f_{2}, f_{1} \in C^{1}, f_{2}$ increasing, we have, from (2.1),

$$
\begin{aligned}
\Delta u+b_{1} u_{1}+f_{1}(u)-f_{1}(0) & =f_{2}(0)-f_{2}(u)-f(0) \\
& \leqq 0
\end{aligned}
$$

and the results follow with the aid of the maximum principle. In particular if $f$ is locally Lipschitz continuous, $f(0) \geqq 0$, and $u \geqq 0, u \neq 0$ in $\Omega$ then the results of Theorem 2.1 hold. If the condition of Lipschitz continuity is weakened to Hölder continuity the results need not hold. For example, if $p>2$, the function $w(x)=\left(1-|x|^{2}\right)^{p}$ in $|x| \leqq 1, w=0$ in $|x|>1$, is in $C^{2}\left(R^{n}\right)$ and satisfies (1.1) with

$$
f(w)=-2 p(p-2) w^{1-2 / p}+2 p(n+2 p-2) w^{1-1 / p} .
$$

The function $f$ is Hölder continuous with exponent $1-2 / p$, and $f(0)=0$. However the function

$$
u(x)=w(x)+w\left(x-x_{0}\right),
$$

with some fixed $x_{0}$ satisfying $\left|x_{0}\right|=3$, satisfies (1.1) in $|x|<5$ with the same $f$. Obviously $u$ does not satisfy the conclusions of Theorem 1 .

2.4. Theorem 2.1 extends to a class of nonlinear elliptic second order equations. With $\Omega, \Sigma(\lambda)$ etc. as before, consider a $C^{2}$ solution $u$ in $\bar{\Omega}$ of such an equation

$$
F\left(x, u, u_{1}, \ldots, u_{n}, u_{11}, \ldots, u_{n n}\right)=0
$$

which is elliptic, i.e., for positive constants $m, M$

$$
M|\xi|^{2} \geqq F_{u_{i j}} \xi_{i} \xi_{j} \geqq m|\xi|^{2} .
$$

The function $F\left(x, u, p_{i}, p_{j k}\right)$ is assumed to be continuous and to have continuous first derivatives with respect to $u, p_{i}$ and $p_{j k}$ for all values of these last arguments, and $x \in \bar{\Omega}$, and to satisfy the following conditions:

(b) On $\partial \Omega \cap\left\{x_{1}>\lambda_{1}\right\}$ the function $g(x)=F(x, 0, \ldots, 0)$ satisfies

$$
g(x) \geqq 0 \forall x, \text { or } \quad g(x)<0 \forall x .
$$

(c) For every $\lambda$ in $\lambda_{1} \leqq \lambda<\lambda_{0}$ and for $x \in \sum(\lambda)$, and all values of the arguments $u$, $p_{j}, p_{j k}$ with $u>0, p_{1}<0$,

$$
F\left(x^{\prime}, u,-p_{1}, p_{2}, \ldots, p_{n}, p_{11},-p_{1 x}, \ldots, p_{\beta \gamma}\right) \geqq F\left(x, u, p_{1}, p_{\alpha}, p_{i j}\right) .
$$

Here $i, j$ range from 1 to $n$ and $\alpha, \beta, \gamma$ from 2 to $n$. Note that condition (b) is automatic in case $F$ is independent of $x$. Furthermore, in the first case in condition (b), it follows from condition (c) that $g(x) \geqq 0$ for $x$ in a neighbourhood in $\Omega$ of $\partial \Omega \cap\left\{x_{1}>\lambda_{1}\right\}$ - because $g(x)$ is a decreasing function of $x_{1}$ there. 
Theorem 2.1'. Let $u$ satisfy condition (a), and F satisfy conditions (b) and (c). Then (2.2) holds, and $u_{1}<0$ in $\Sigma$. Furthermore if $u_{1}=0$ at some point in $\Omega$ on $T_{\lambda_{1}}$ then, necessarily, $u$ is symmetric in the plane $T_{\lambda_{1}}$ and (2.3) holds.

The proof of this is exactly the same as that of Theorem 2.1, in particular Lemmas 2.1, 2.2 hold, and is left to the reader. As an immediate application we have the following symmetry result:

Corollary 1. With $\Omega$ as before, suppose that $\lambda_{1}=0$, and that $\Omega$ is symmetric about the plane $x_{1}=0$. Suppose our solution $u>0$ in $\Omega, u=0$ on $\partial \Omega$. Assume $F$ satisfies condition (b) and, in place of condition (c), conditions

$\left(\mathrm{c}_{1}^{\prime}\right) F$ is symmetric in $x_{1}$, and decreasing in $x_{1}$ for $x_{1}>0$.

$\left(c_{2}^{\prime}\right) F\left(x, u,-p_{1}, p_{x}, p_{11},-p_{1 x}, p_{\beta \gamma}\right) \equiv F\left(x, u, p_{1}, p_{\alpha}, p_{i j}\right)$ if $u>0$.

Then $u$ is symmetric in $x_{1}$ and $u_{x_{1}}<0$ for $x_{1}>0$.

Proof. Conditions $\left(\mathrm{c}_{1}^{\prime}\right),\left(\mathrm{c}_{2}^{\prime}\right)$ imply condition (c) since, by $\left(\mathrm{c}_{1}^{\prime}\right)$, for $\lambda_{1} \leqq \lambda$, the lefthand side of $(2.6) \geqq$ the left-hand side of $\left(\mathrm{c}_{2}^{\prime}\right)$. By Theorem $2.1^{\prime}$ we find

$$
u_{1}(x)<0 \quad \text { and } \quad u\left(-x_{1}, x^{\prime}\right) \geqq u\left(x_{1}, x^{\prime}\right) \text { for } \quad x_{1}>0 .
$$

If we replace $x_{1}$ by $-x_{1}$, i.e., reverse the $x_{1}$-axis, we may apply the theorem again and obtain just the opposite inequalities. Here $x^{\prime}=\left(x_{2}, \ldots, x_{n}\right)$.

2.5. We also obtain generalizations of Theorems 1 and 2 .

Theorem 1'. Theorems 1 and 2 hold for $f=f(r, u)$ depending also on $r$, with $f, f_{u}$ continuous, provided $f$ is decreasing in $r$.

One might ask whether positive solutions $u$ in a ball $|x| \leqq R$, vanishing on the boundary, of

$$
\Delta u+f(r, u)=0
$$

are necessarily spherically symmetric - even if $f$ is not decreasing in $r$. This is not the case in general. For example, let $w$ be an eigenfunction of

$$
\Delta w+\lambda w=0, \quad w=0 \quad \text { on } \quad|x|=R,
$$

which is not spherically symmetric. Then for $\varepsilon>0$ small, the function $u=R^{2}-|x|^{2}$ $+\varepsilon w$, is positive in $|x|<R$, vanishes on the boundary and satisfies

$$
\Delta u+\lambda u+\lambda\left(r^{2}-R^{2}\right)+2 n=0 \quad \text { in } \quad|x|<R ;
$$

but $u$ is not spherically symmetric.

Using Theorem $2.1^{\prime}$ one may prove further symmetries. The following, whose proof is left to the reader, is an example. See also Remark 2 at the end of Sect. 3. Let $\Omega$ be the unit ball in $\mathbb{R}^{2 n}$ and denote the points in $\mathbb{R}^{2 n}$ by $(x, y), x, y \in \mathbb{R}^{n}$.

Corollary 2. Let $u \in C^{2}(\bar{\Omega}), u>0$ in $\Omega$, be a solution of

$$
\Delta u+f(x, y, u)=0 \quad \text { in } \quad \Omega, \quad u=0 \quad \text { on } \quad \partial \Omega .
$$


Assume $f$ and $f_{u}$ are continuous in $\bar{\Omega} \times\{u \geqq 0\}$ and

(i) $f(x, y, 0) \geqq 0$ everywhere on $\partial \Omega$, or $f(x, y, 0)<0$ everywhere on $\partial \Omega$.

(ii) $f(x, y, u)=f(y, x, u)$.

(iii) For every point $(x, x) \in \Omega$ and for every vector $z \in \mathbb{R}^{n}$ and $\forall u>0$, the function $f(x+s z, x-s z, u)$

as a function of $s>0$ is nonincreasing (wherever defined).

Then $u(x, y)=u(y, x)$ and for $x, z \in \mathbb{R}^{n},|x|<1 / \sqrt{2}$

$\frac{d}{d s} u(x+s z, x-s z)<0$

for

$$
0<s<\frac{1}{|z|} \sqrt{\frac{1}{2}-|x|^{2}} .
$$

The function $f(x, y, u)=g(|x-y|, u)$ with $g(t, u)$ decreasing in $t$, for $t>0$, satisfies the conditions (ii) and (iii).

Theorem 1' admits extension to still more general rotationally symmetric equations. In particular, if $\Omega$ is rotationally symmetric with respect to some of the $x$ variables, say $\left(x_{1}, \ldots, x_{k}\right)$ and the Eq. $\left(2.1^{\prime}\right)$ also has this property, then one may prove an extension of Theorem 2.1' showing, under suitable hypotheses, that $u$ is a function of $\left(\varrho, x_{k+1}, \ldots, x_{n}\right)$ for $\varrho=\sum_{1}^{k} x_{g}^{2}$, and $u_{\varrho}<0$ for $\varrho>0$.

2.6. We also have the following extension of Theorem 3.

Theorem 3'. Let $u$ be as in Theorem 3 but satisfying a more general equation than (1.3):

$$
\ddot{u}+f(x, u, \dot{u})=0 \quad \text { on } \quad 0<x \leqq 1 .
$$

Here $f(x, u, p), f_{u}$ and $f_{p}$ are continuous. Then $\dot{u}(x)<0$ for $\frac{1}{2}<x<1$ provided $f$ satisfies

$$
f(y, u,-p) \geqq f(x, u, p)
$$

whenever $u>u(1), p \leqq 0$, and $y+x>1, y<x$. Furthermore if $\dot{u}\left(\frac{1}{2}\right)=0$ then $u$ is symmetric about $x=\frac{1}{2}$.

The following is a simple consequence:

Theorem $3^{\prime \prime}$. Let $u \in C^{2}\left(R^{1}\right)$ be a positive function satisfying

$$
\ddot{u}+f(u, \dot{u})=0 \quad \text { on } R^{1}
$$

with $f \in C^{1}$ and $f(u, p)=f(u,-p)$ for $u>0$. If $u(x) \rightarrow 0$ as $x \rightarrow \pm \infty$ and $u$ assumes its maximum at the origin, then $u$ is symmetric in $x$ and $\dot{u}(x)<0$ for $x>0$.

2.7. Here is another application of Theorem 2.1 with a novel conclusion.

Corollary 3. Let $\Omega$ be a convex domain which is close to a ball $|x|<R$ in the sense that their boundaries are close in the $C^{2}$ topology. In $\Omega$ let $u$ be a positive solution of

$$
\Delta u+f(u)=0, \quad u=0 \quad \text { on } \quad \partial \Omega .
$$


Then all stationary points of $u$ in $\Omega$, in particular wherever $u$ takes its maximum, lie in a small neighbourhood of the origin.

Proof. According to Theorem $2.1 u$ has no stationary point in any maximal cap. Their union covers all of $\Omega$ except for a small region about the origin.

This result suggests the following:

Problem: Suppose $u>0$ is a solution of (2.7) in a bounded domain $\Omega$ in $\mathbb{R}^{n}, u=0$ on $\partial \Omega$, say $u \in C^{2}(\bar{\Omega})$. Is there some $\varepsilon>0$ depending only on $\Omega$ (i.e., independent of $f$ and $u$ ) such that $u$ has no stationary point in an $\varepsilon$-neighbourhood of $\partial \Omega$ ?

This is true for $n=2$ in case $f(u) \geqq 0$ for $u \geqq 0$, but for $n>2$ the problem is open.

Proof. Given any boundary point $x_{0}$ of $\Omega$ we will show that there is a neighbourhood of it in $\Omega$, determined solely by the geometry, which contains no stationary point of $u$. The desired result then follows. Let $D$ be a closed disc touching $\bar{\Omega}$ only at the point $x_{0}$. For convenience we suppose it is the unit disc with centre at the origin.

We perform a reflection in the unit circle

$$
x \mapsto y=\frac{x}{|x|^{2}}
$$

and set

$$
u(x)=v(y) .
$$

The image $\tilde{\Omega}$ of $\Omega$, lies inside $D$, and $\overline{\tilde{\Omega}}$ touches the boundary only at $x_{0}$. A simple calculation shows that in $\tilde{\Omega}, v(y)$ satisfies

$$
\Delta v+|y|^{-4} f(v)=0
$$

for $n>2$ the equation has additional terms.

Let $\Sigma=\Sigma_{x_{0}}$ be the maximal cap of $\tilde{\Omega}$ corresponding to the direction $x_{0}$ which we may take to be $(1,0)$. Since $\tilde{\Omega}$ is strictly convex near $x_{0}, \Sigma$ contains a full neighbourhood of $x_{0}$ in $\tilde{\Omega}$. Since $f \geqq 0$ for $u \geqq 0$, we see that condition (c) of Theorem $2.1^{\prime}$ is satisfied. We may therefore apply the theorem and infer that $\operatorname{grad} v \neq 0$ in $\Sigma$. Hence $\operatorname{grad} u \neq 0$ in the image of $\Sigma$ under the reflection in the unit circle. This image contains a full neighbourhood of $x_{0}$ in $\Omega$, in fact it is $\Omega \cap\{\mathrm{a}$ circle passing through the origin with some radius $>1\}$, and the proof is finished.

As a direct application of this and Theorem 2, whose details we leave to the reader, we have:

Example. Suppose $u$ is a positive solution of (here $f$ is locally Lipschitz):

$$
\Delta u+f(u)=0 \quad \text { in } \quad R^{\prime} \leqq|x| \leqq R, \quad \text { in } \mathbb{R}^{2},
$$

which vanishes on the boundary. Suppose $f(u) \geqq 0$ for $u \geqq 0$. Then all the critical points of $u$ are in the region

$$
\frac{2 R^{\prime} R}{R^{\prime}+R}<|x|<-\frac{R^{\prime}+R}{2} .
$$


Remark $1^{\prime}$. As in Remark 1, the results of Theorem 2.1', Corollary 1 (Theorems 1', $3^{\prime}$ and Corollaries 2, 3 respectively) hold if $F=F_{1}+F_{2}\left(f=f_{1}+f_{2}\right.$ respectively), where $F_{1}\left(f_{1}\right)$ has continuous first derivatives with respect to $u, p_{i}, p_{i k}$, and $F_{2}\left(f_{2}\right)$ has continuous first derivatives with respect to $p_{i}, p_{j k}$, and $F_{2}$ is monotone increasing in $u$; both $F_{1}, F_{2}$ are to satisfy conditions (b) and (c).

2.8. Theorem 1 yields a positive response to a question put us by C. Holland. For $p>1$, is the positive solution $u$ of

$$
\Delta u+u^{p}=0 \quad \text { in }|x|<R, \quad u=0 \quad \text { on }|x|=R
$$

unique? (The question is still open for other domains.) According to Theorem 1 the solution is spherically symmetric, and so satisfies

$$
u_{r r}+\frac{n-1}{r} u_{r}+u^{p}=0, \quad 0<r<R, \quad u_{r}(0)=0,
$$

with $u(R)=0$, and $u_{r}(r)<0$ for $r>0$. We use

Lemma 2.3. Let $u$ and $v$ be positive solutions of (2.9). For $i^{2 /(p-1)}=u(0) / v(0)$, $u(r)=\lambda^{2 /(p-1)} v(\lambda r)$.

Proof. The function $w(r) \equiv \lambda^{2 /(p-1)} v(\lambda r)$ is also a solution of (2.9) and at $r=0$ it agrees with $u(0)$.

As solutions of the elliptic Eq. (2.8), $u$ and $w$ are analytic, i.e., they are analytic functions of $r^{2}$. But in fact all their derivatives at $r=0$ may be computed in terms of $u(0)$ showing that $w \equiv u$. For example if we let $r \rightarrow 0$ in (2.9) we find

$$
n u_{r r}(0)+u^{p}(0)=0 \text {. }
$$

By further differentiation we may compute all the derivatives, and the lemma is proved.

From the lemma it follows easily that the positive solution of (2.9) vanishing at $R$ is unique.

\section{Further Results}

3.1. In this section we take up extensions of results of Sect. 2 to optimal caps and to special domains with corners. We shall use the same notation.

We first try to extend Theorem $2.1^{\prime}$ to optimal caps. Consider a solution $u$ of (2.1') with $F$ independent of $u_{1 x}$ for $\alpha>1$ :

$$
F\left(x, u, u_{j}, u_{11}, u_{\alpha \beta}\right)=0 ;
$$

here $j$ ranges from 1 to $n$ and $\alpha, \beta$ from 2 to $n$. This includes Eq. (2.1).

Assume that $u$ satisfies, in place of (a) of Sect. 2.1:

(A) $u \in C^{2}(\bar{\Omega}), u>0$ in $\Omega, u=0$ on $\partial \Omega$.

We assume that $F$ satisfies conditions (b), (c) as before but in an optimal cap:

(B) On $\partial \Omega \cap\left\{x_{1}>\lambda_{2}\right\}$ the function $g(x)=F(x, 0, \ldots, 0)$ satisfies

$$
g(x) \geqq 0 \forall x, \quad \text { or } \quad g(x)<0 \forall x .
$$


(C) For every $\lambda$ in $\lambda_{2} \leqq \lambda<\lambda_{0}$ and for $x \in \Sigma(\lambda)$ and all values of the arguments $u, p_{j}, p_{j k}$ with $u>0, p_{1}<0$,

$$
F\left(x^{\lambda}, u,-p_{1}, p_{2}, \ldots, p_{n}, p_{11}, p_{\alpha \beta}\right) \geqq F\left(x, u, p_{1}, \ldots, p_{n}, p_{\alpha \beta}\right) .
$$

Theorem 3.1. Assume conditions (A), (B) and (C) are satisfied. Then for $\lambda_{2}<\lambda_{<}<\lambda_{0}$,

$$
u_{1}(x)<0 \text { and } u(x)<u\left(x^{\lambda}\right) \text { for } x \in \Sigma(\lambda) .
$$

Furthermore, if $u_{1}=0$ at some point on $\Omega \cap T_{\lambda_{2}}$, then $u$ is symmetric in the plane $T_{\lambda_{2}}$ and

$$
\Omega=\Sigma\left(\lambda_{2}\right) \cup \Sigma^{\prime}\left(\lambda_{2}\right) \cup\left(T_{\lambda_{2}} \cap \Omega\right) .
$$

Proof of Theorem 3.1. The proof begins like that of Theorem 2.1. By Lemma 2.1 (see Sect. 2.4), for $\lambda$ close to $\lambda_{0}, \lambda<\lambda_{0}$, we find that (3.3) holds.

Decrease $\lambda$ until a critical value $\mu \geqq \lambda_{2}$ is reached beyond which it no longer holds. Then for $\lambda=\mu$ we have

$$
u_{1}(x)<0 \text { and } u(x) \leqq u\left(x^{\mu}\right) \text { for } x \in \Sigma(\mu) .
$$

We will show that $\mu=\lambda_{2}$. Suppose $\mu>\lambda_{2}$. For some point $x_{0} \in \partial \Sigma(\mu) \backslash T_{\mu}$ we have $x_{0}^{\mu} \in \Omega$. Since $0=u\left(x_{0}\right)<u\left(x_{0}^{\mu}\right)$ we see that $u(x) \neq u\left(x^{\mu}\right)$ in $\Sigma(\mu)$. In $\Sigma^{\prime}(\mu)$ we set

$$
v(x)=u\left(x^{\mu}\right), \quad w(x)=v(x)-u(x)
$$

so that $w \leqq 0, w \neq 0$. The function $v$ satisfies, $v_{1}>0$ and

$$
\begin{aligned}
& F\left(x, v(x), v_{j}, v_{11}, v_{\alpha \beta}\right) \\
& \quad=F\left(x, u\left(x^{\mu}\right),-u_{1}\left(x^{\mu}\right), u_{\gamma}\left(x^{\mu}\right), u_{11}\left(x^{\mu}\right), u_{\alpha \beta}\left(x^{\mu}\right)\right) .
\end{aligned}
$$

Since $x^{\mu} \in \Sigma(\mu)$ and $u_{1}\left(x^{\mu}\right)<0$ we see from (3.2) that the last expression is

$$
\begin{aligned}
& \geqq F\left(x^{\mu}, u\left(x^{\mu}\right), u_{j}\left(x^{\mu}\right), u_{11}\left(x^{\mu}\right), u_{\alpha \beta}\left(x^{\mu}\right)\right) \\
& =0 .
\end{aligned}
$$

Using $F(x, u, \ldots)=0$ in $\Sigma^{\prime}(\mu)$ we may apply the theorem of the mean in integral form and conlcude that $w$ satisfies a differential inequality of the form (1.10) in $\Sigma^{\prime}(\mu)$ with $a_{1 x}=0$ for $\alpha>1$.

By the maximum principle, $w<0$ in $\Sigma^{\prime}(\mu)$, and by Lemma $H$, since $w$ achieves its maximum, zero, at every point of $T_{\mu}$,

$$
0<w_{1}=-2 u_{1} \quad \text { on } \quad T_{\mu} \cap \Omega .
$$

Thus

$$
u(x)<u\left(x^{\mu}\right) \text { for } x \in \Sigma(\mu) \text { and } u_{1}<0 \text { on } \Omega \cap T_{\mu} ;
$$

in particular (3.3) holds for $\lambda=\mu$. Furthermore, by Lemma $S$, at any point $Q \in \hat{\partial} \Omega \cap T_{\mu}$ where $T_{\mu}$ is orthogonal to $\partial \Omega$,

$$
\frac{\partial w}{\partial s}<0 \text { or } \frac{\partial^{2} w}{\partial s^{2}}<0 \text { at } Q
$$


for any direction $s$ at $Q$ entering $\Sigma^{\prime}(\mu)$ nontangentially. At $Q$ however the functions $v$ and $u$ have the same normal derivative to $\partial \Omega$ and zero tangential derivatives, so that $\partial w(Q) / \partial s=0$. Hence

$$
\frac{\partial^{2} w}{\partial s^{2}}(Q)<0 \text {. }
$$

From our definition of $\mu$ one of the following holds:

(i) There is a sequence $y_{j}$ converging to some point $y$ on $T_{\mu}$ with

$u_{1}\left(y_{j}\right) \geqq 0$

$O r^{*}$

(ii) There are sequences $\lambda^{j}, \lambda_{2}<\lambda^{j} \nearrow \mu$, and $x_{j} \in \Sigma\left(\lambda^{j}\right)$ such that

$$
u\left(x_{j}\right) \geqq u\left(x_{j}^{\lambda \prime}\right) .
$$

Consider case (i). Clearly $u_{1}(y) \geqq 0$. By (3.9), $y$ must lie on $\partial \Omega$. Suppose $T_{\mu}$ is not orthogonal to $\partial \Omega$ at $y$. Then necessarily $v_{1}(y)>0$, and by Lemma 2.1, $u_{1}<0$ in a neighbourhood in $\Omega$ of $y$-a contradiction. Thus $T_{\mu}$ must be orthogonal to $\partial \Omega$ at $y$; we may suppose $v(y)=(0, \ldots, 0,1)=e_{n}$. Choose the direction $s$ to be $(-1 / \sqrt{2}, 0, \ldots, 0,-1 / \sqrt{2})$. Then according to $(3.10)$

$$
\left(\frac{\partial}{\partial x_{1}}+\frac{\partial}{\partial x_{n}}\right)^{2} w<0 \quad \text { at } y
$$

i.e.,

$$
\left(\frac{\partial}{\partial x_{1}}+\frac{\hat{\partial}}{\partial x_{n}}\right)^{2} v<\left(\frac{\hat{\partial}}{\partial x_{1}}+\frac{\partial}{\partial x_{n}}\right)^{2} u \text { at } y .
$$

But $v_{11}=u_{11}, v_{n n}=u_{n n}, v_{1 n}=-u_{1 n}$ at $y$, so

$$
u_{1 n}>0 \text { at } y \text { and hence near } y \text {. }
$$

If we integrate this on the segment in the $e_{n}$ direction from $y_{j}(j$ large), to the point $x$ where it hits $\partial \Omega$ we find that $u_{1}(x)>0$. But for $x \in \overline{\Sigma\left(\hat{\lambda}_{2}\right)} \cap \partial \Omega$ we have $v_{1}(x) \geqq 0$ and hence $u_{1}(x) \leqq 0$. Thus case (i) is impossible.

On to case (ii). We may suppose $x_{j}$ converges to $x$ in $\overline{\Sigma(\mu)}$; then $u(x) \geqq u\left(x^{\mu}\right)$. By (3.9), $x \in \partial \Sigma(\mu)$. If $x$ lies on $T_{\mu}$ then for $j$ large the segment from $x_{j}$ to $x_{j}^{\hat{\lambda}}$ lies in $\Omega$, and then contains a point $y_{j}$ satisfying the conditions of case (i) - which we know to be impossible. Thus $x \notin T_{\mu}$. If $x^{\mu} \in \Omega$ then $0=u(x)<u\left(x^{\mu}\right)$, a contradiction, so we also have $x^{\mu} \in \partial \Omega$. Since $\mu>\lambda_{2}$ it follows that $v(x)=v\left(x^{\mu}\right)$ and these are orthogonal to $(1,0, \ldots, 0)$. We may suppose these normals are $e_{n}=(0, \ldots, 0,1)$. But then in $\overline{\Sigma^{\prime}(\mu)}$ the function $w(x)$ defined in (3.8) achieves its maximum, zero, at $x^{\mu}$ and hence by Lemma $H$

$$
w_{n}\left(x^{\mu}\right)>0 \text {. }
$$

On the other hand, since $\Sigma_{\sim}^{\prime}\left(\lambda_{j}\right) \subset \Omega$, the segment $I_{j}$ in the $e_{n}$ direction from $x_{j}$ to $\partial \Omega$ is not longer than that, $\tilde{I}_{j}$, from $x_{j}^{\hat{\lambda}^{j}}$ to $\partial \Omega$. Using the fact that $u=0$ on $\partial \Omega$ it follows from the theorem of the mean that $I_{j}$ and $\tilde{I}_{j}$ contain points $z_{j}, \tilde{z}_{j}$ respectively 
such that

$$
\begin{aligned}
-u\left(x_{j}\right) & =u_{n}\left(z_{j}\right)\left|I_{j}\right| \\
-u\left(x_{j}^{\lambda^{J}}\right) & =u_{n}\left(\tilde{z}_{j}\right)\left|\tilde{I}_{j}\right| .
\end{aligned}
$$

Here $\left|I_{j}\right|$ denotes the length of $I_{j}$. Hence

$$
-u_{n}\left(z_{j}\right)\left|I_{j}\right| \geqq-u_{n}\left(\tilde{z}_{j}\right)\left|\tilde{I}_{j}\right|>0
$$

and since $\left|I_{j}\right| \leqq\left|\tilde{I}_{j}\right|$,

$$
-u_{n}\left(z_{j}\right) \geqq-u_{n}\left(\tilde{z}_{j}\right) \text {. }
$$

Letting $j \rightarrow \infty$ we find

$$
-u_{n}(x) \geqq-u_{n}\left(x^{\mu}\right),
$$

contradicting (3.12). Thus case (ii) is impossible.

We have proved that $\mu=\lambda_{2}$, and hence (3.3) for $\lambda_{2}<\lambda<\lambda_{0}$. The remainder of the proof of the theorem is the same as for Theorem 2.1 and will be omitted.

Theorem 3.1 admits various applications as in Sect. 2. We mention only one:

Corollary 1. Let $\Omega$ be symmetric about $x_{1}=0$ and convex in the $x_{1}$ direction. Suppose $u \in C^{2}(\bar{\Omega})$ is a solution of

$$
\Delta u+f(x, u)=0 \text { in } \Omega,
$$

satisfying condition (A). Assume $f$ and $f_{u}$ are continuous for $x \in \bar{\Omega}$, and $f$ is symmetric in $x_{1}$ with $f$ decreasing in $x_{1}$ for $x_{1}>0$. Then $u$ is symmetric in $x_{1}$ and $u_{x_{1}}<0$ for $x_{1}>0$.

3.2. Our results have required smoothness of $\partial \Omega$. Next we consider a special domain with corners, namely a finite cylinder: $\Omega=(-a, a) \times G$ where $G$ is a bounded domain in $R^{n-1}$ with $\partial G$ smooth. (It will be clear that this result can be extended to more general situations.) Corresponding to $\gamma=e_{1}$ we have $\lambda_{2}=0$, i.e., the optimal cap is $\Omega \cap\left\{x_{1}>0\right\}$. We consider a solution $u$ in $\Omega$ of (3.1) satisfying

$$
\text { (A') } u \in C^{2}(\bar{\Omega}), \quad u>0 \text { in } \Omega, \quad u=0 \text { on } \partial \Omega \text {. }
$$

The function $F$ in (3.1) is assumed to be independent of $u_{1}$, i.e., (3.1) has the form $F\left(x, u, u_{2}, \ldots, u_{n}, u_{11}, u_{\beta \gamma}\right)=0$.

Also $F$ is to satisfy condition (C) which now just takes the form:

(C') $F$ is decreasing in $x_{1}$ for $x_{1}>0$.

We do not require condition (B).

Theorem 3.2. Under the preceding conditions on $u$ and $F$ the results of Theorem 3.1 hold.

Proof. It is the same as that of Theorem 3.1 except at the very beginning, when we assert that for $\lambda$ less than, but close to, $\lambda_{0}$ conditions (3.3) hold. We cannot rely here on Lemma 2.1 since the boundary is not smooth. To get around this difficulty we will use the corollary of the maximum principle of $\S 1$ to prove: 
Lemma 3.1. For $\lambda$ less than but close to $\lambda_{0},(3.3)$ holds.

Proof. For $\lambda_{0}-\varepsilon<\lambda<\lambda_{0}$ the region $\Sigma^{\prime}(\lambda)$ has width $<\varepsilon$ in the direction $e_{1}=(1,0, \ldots, 0)$. In $\Sigma^{\prime}(\lambda)$ the function $v(x)=u\left(x^{\lambda}\right)$ satisfies

$$
F\left(x, v(x), v_{\alpha}(x), v_{11}(x), v_{\beta \gamma}(x)\right) \geqq F\left(x^{\lambda}, v(x), v_{\alpha}(x), v_{11}(x), v_{\beta \gamma}(x)\right)=0 .
$$

Hence $w=v(x)-u(x)$ satisfies an inequality of the form (1.10) with bounded coefficients in $\Sigma^{\prime}(\lambda)$. Also $w \leqq 0$ on $\partial \Sigma^{\prime}(\lambda)$. It follows from the maximum principle and its corollary that for $\varepsilon$ small, $w<0$ in $\Sigma^{\prime}(\lambda)$; by Lemma $\mathrm{H}$, on $T_{\lambda}$, $0<w_{1}=-2 u_{1}$. Consequently (3.3) holds for $\lambda$ close to $\lambda_{0}$. Q.e.d.

Theorems 3 and $3^{\prime}$ are essentially special cases of Theorem 3.2.

Using the same argument one proves the following results.

Theorem 3.2'. Let $\Omega$ be an isosceles triangle in the plane with base on the $x$ axis, centered at the origin. Let $u \in C^{2}(\bar{\Omega} \backslash$ corners $) \cap C(\bar{\Omega})$ satisfy

$$
\Delta u+f(u)=0 \quad \text { in } \Omega, \quad u=0 \quad \text { on } \partial \Omega,
$$

with flocally Lipschitz continuous. If $u>0$ in $\Omega$, then $u$ is symmetric about the $y$ axis, and $u_{x}<0$ for $x>0$ in $\Omega$.

Theorem 3.2". Let $u$ and $f$ he as in Theorem 3.2', but with $\Omega$ an infinite angular sector $0<\theta<\theta_{0}<\pi$. Then for any fixed angle $\phi$ in $\theta_{0}-\pi / 2<\phi<\pi / 2, u_{x} \cos \phi+u_{y} \sin \phi>0$ at every point of $\Omega$.

Both results admit various extensions to higher dimensions.

3.3. Let us specialize this still further and suppose that $G$ is a ball $\left|x^{\prime}\right|<b$ in $R^{n-1}$; here $x^{\prime}=\left(x_{2}, \ldots, x_{n}\right)$, and that the equation has rotational symmetry in $G$.

Can we conclude the same of the solution? We will take up a simple case : $u>0$ is a solution in $\Omega$ of

$$
a_{11}\left(x_{1}\right) u_{11}+\sum_{\alpha>1} u_{z \alpha}+f\left(x_{1},\left|x^{\prime}\right|, u\right)=0
$$

with $u \in C^{2}(\bar{\Omega}), u=0$ on $\partial \Omega$ and $f, f_{u}$ continuous in $\bar{\Omega} \times R^{+}$. Assume conditions (B) and $(\mathrm{C})$ in the following form:

(苂) $f\left(x_{1}, b, 0\right) \geqq 0$ for $\left|x_{1}\right| \leqq a$

or

$$
f\left(x_{1}, b, 0\right)<0 \text { for }\left|x_{1}\right| \leqq a .
$$

(更) $f\left(x_{1}, \varrho, u\right)$ is decreasing in $\varrho$ for $0<\varrho<b$.

Theorem 3.3. Under the conditions above, $u$ is radially symmetric in the variables $x^{\prime}$, i.e., $u=u\left(x_{1},\left|x^{\prime}\right|\right)$, and

$$
u_{\varrho}\left(x_{1}, \varrho\right)<0 \text { for }\left|x_{1}\right|<a, \quad 0<\varrho<b \text {. }
$$

The proof uses the analogue of Lemma 2.1:

Lemma 3.2. The set $\sum=\left\{\left|x_{1}\right| \leqq a\right\} \times\left(\partial G \cap\left\{x_{n}>0\right\}\right)$ has a neighbourhood in $\Omega$ in which $u_{n}<0$. 
Proof. Lemma 2.1 (with the variable $x_{n}$ in place of $x_{1}$ ) applies to any point $x_{0} \in\left\{x_{1}<a\right\} \times\left(\partial G \cap\left\{x_{n}>0\right\}\right)$ and gives the desired result. Thus we need only consider a point $x_{0}$ in $\Sigma$ with $x_{01}= \pm a$; suppose $x_{01}=a$. So

$$
x_{0}=\left(a, x_{02}, \ldots, x_{0 n}\right), \quad \sum_{2}^{n} x_{0 j}^{2}=b^{2}, \quad x_{0 n}>0 .
$$

Consider the first case in $(\tilde{\mathrm{B}})$. By $(\tilde{\mathrm{C}})$, for $x=\left(x_{1}, x^{\prime}\right)$ close to $x_{0}$, we have

$$
f\left(x_{1},\left|x^{\prime}\right|, 0\right) \geqq 0
$$

Consequently by the theorem of the mean we see that in such a neighbourhood of $x_{0}, u$ satisfies

$$
a_{11} u_{11}+\sum_{\alpha>1} u_{\alpha \alpha}+c(x) u \leqq 0
$$

for some continuous function $c$. We may therefore apply Lemma $\mathrm{S}$ to $-u$ and conclude, since $\operatorname{grad} u\left(x_{0}\right)=0$, that $\left(\partial_{1}+\partial_{n}\right)^{2} u>0$ at $x_{0}$. Since $u_{11}=u_{n n}=0$ there we have

$$
u_{1 n}\left(x_{0}\right)>0 \text {. }
$$

Hence $u_{1 n}>0$ near $x_{0}$ and since $u_{n}=0$ on $x_{1}=a$ the desired conclusion follows.

In the second case of $(\tilde{\mathrm{B}})$ we have $a_{11} u_{11}+\sum_{\alpha>1} u_{\alpha \alpha}>0$ at $x_{0}$. But in fact this expression is zero at $x_{0}$ so that the case does not apply. The lemma is proved.

Proof of Theorem 3.3. We will prove $u$ is symmetric in $x_{n}$ and $u_{n}<0$ for $x_{n}>0$. Since we may rotate the coordinates $x^{\prime}$ the general result will follow. Using Lemma 3.1, we see that for $\lambda<b$ but $\lambda$ close to $b$, the analogue of (3.3) holds, i.e.,

$$
u_{n}(x)<0 \text { and } u(x)<u\left(x^{\hat{\lambda}}\right) \text { for } \lambda<x_{n}<b \text {; }
$$

here $x^{\lambda}$ is the reflection of $x$ in the plane $x_{n}=\lambda$. It suffices to prove that $\overline{(3.3)}$ holds for every $\lambda>0$. To do this we proceed as in the proof of Theorem 3.1. Decrease $\lambda$ until a critical value $\mu \geqq 0$ is reached beyond which $(\overline{3.3)}$ no longer holds. Then we have

$$
u_{n}(x)<0 \quad \text { and } \quad u(x) \leqq u\left(x^{\mu}\right) \text { for } x_{n}>\mu .
$$

We wish to show that $\mu=0$. Suppose $\mu>0$. We follow the proof of Theorem 3.1, and have to consider cases (i) or (ii) there (with $x_{n}$ in place of $x_{1}$ ). In case (i) we also have to look at the possibility that $y$ lies on $T_{\mu} \cap\{\partial G \times[-a, a]\}$. This is excluded by Lemma 3.2. Case (ii) is treated as before and we may regard the proof as complete.

Remark 1 . All the preceding results remain valid if we replace $F$ (respectively $f$ ) by $F_{1}+F_{2}\left(f_{1}+f_{2}\right)$ where $F_{1}, F_{2}\left(f_{1}, f_{2}\right)$ are as in Remark 1' in Sect. 2 - under the additional condition (3.1).

3.4. We conclude with an analogue of Corollary 2 of Theorem 2.1'. 
Remark 2. Let $\Omega=(-1,1) \times(-1,1)$ in $\mathbb{R}^{2}$ and let $u(x, y)$ be a positive solution in $\Omega$ belonging to $C^{2}(\bar{\Omega})$ of

$$
\Delta u+f(x, y, u)=0 \quad \text { in } \quad \Omega, \quad u=0 \quad \text { on } \quad \partial \Omega
$$

where $f, f_{u}$ are continuous in $\bar{\Omega} \times R^{+}$. Assume $f$ satisfies

(i) $f$ is symmetric in $x$ and $y$ and nonincreasing on each segment $S$ perpendicular to the diagonal $D: x=y$ as we go from the diagonal to $\partial \Omega$

(ii) $f(x, y, 0) \geqq 0$ for all $(x, y) \in \partial \Omega$ or $f(x, y, 0)<0$ for all $(x, y) \in \partial \Omega$.

Then $u$ is symmetric in $x$ and $y$ and is strictly decreasing on each such interval $S$.

Proof. For convenience we set $\Omega$ as in the figure so that $f(x, y, u)$ is symmetric in $x$ and nonincreasing in $x$ for $x>0$.

The proof is then identical to that of Theorem 2.1, moving lines $x=\lambda$, once we can get it started, i.e., for $\lambda$ less than but close to $1 / \sqrt{2}$.

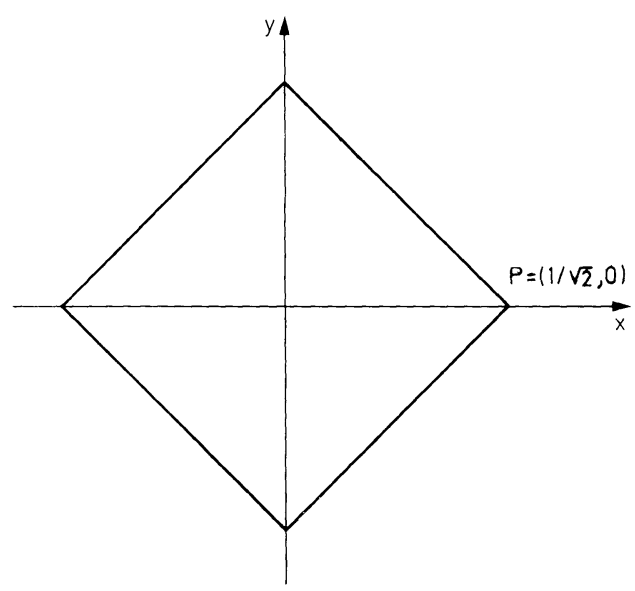

Fig. 3

We have only to show that $u_{1}<0$ in a neighbourhood in $\Omega$ of $P$, and the proof then proceeds as before. This is proved as in the proof of Theorem 3.1 using Lemmas $\mathrm{H}$ and $\mathrm{S}$.

\section{The Proof of Theorem 4}

First, the

Proof of Proposition 1. Under the change of variables

$$
x \rightarrow y=\frac{x}{|x|^{2}}, \quad v(x)=|y|^{n-2} u(y)
$$


The Eq. $\left(1.8^{\prime}\right)$ transforms to the equation in $y \neq 0$

$$
\Delta u(y)=-|y|^{-n-2} f\left(|y|^{n-2} u(y)\right)=-F(y)
$$

and $u(y)$ is bounded near $y=0$. Furthermore

$$
F(y)=|y|^{-n-2} f\left(|y|^{n-2} u(y)\right)=u^{k} g\left(|y|^{n-2} u(y)\right)|y|^{k(n-2)-(n+2)}
$$

is bounded near $y=0$. It is then easy to see that $u$ is a distribution solution of (4.1) in $R^{n}$ including the origin. Since $F(y)$ is bounded near the origin we see that $u \in W^{2, p}, \forall p<\infty$; thus $u \in C^{1}$. But then $F(y)$ is Hölder continuous near the origin and it follows that $u$ has Hölder continuous second derivatives. From the maximum principle it follows that $u(0)=a_{0}>0$. Consequently near the origin we have

$$
u(y)=a_{0}+u_{i}(0) y_{i}+\frac{1}{2} u_{i j}(0) y_{i} y_{j}+o\left(|y|^{2}\right)
$$

and

$$
u_{i}(y)=u_{i}(0)+u_{i j}(0) y_{j}+o(|y|) .
$$

These yield (1.9) for $v(x)=|x|^{2-n} u\left(\frac{x}{|x|^{2}}\right)$.

Proof of Theorem 4. We shall apply the procedure of moving planes from infinity but in order to get it started we first shift the origin in order to simplify the expansions (1.9). Replace $x$ by $x-x_{0}$ where

$$
x_{0 j}=-\frac{a_{j}}{m a_{0}} .
$$

Since, for $q>0$,

$$
\frac{1}{\left|x-x_{0}\right|^{q}}=\frac{1}{|x|^{q}}\left(1+\frac{q}{|x|^{2}} x_{j} x_{0 j}+\ldots\right)
$$

we find

$$
\begin{aligned}
v & =\frac{1}{|x|^{m}}\left(1+\frac{m}{|x|^{2}} x_{j} x_{0 j}+\ldots\right)\left(a_{0}+\frac{a_{j}\left(x_{j}-x_{0 j}\right)}{\left|x-x_{0}\right|^{2}}+\frac{a_{i j} x_{i} x_{j}}{|x|^{4}}+o\left(\frac{1}{|x|^{2}}\right)\right) \\
& =\frac{1}{|x|^{m}}\left(a_{0}+\frac{a_{i j} x_{i} x_{j}}{|x|^{4}}+o\left(\frac{1}{|x|^{2}}\right)\right)
\end{aligned}
$$

with different coefficients $a_{i j}$. Similarly

$$
\begin{aligned}
v_{x_{1}}= & -\frac{m\left(x_{i}-x_{0 i}\right)}{|x|^{m+2}}\left(1+\frac{m+2}{|x|^{2}} x_{k} x_{0 k}+\ldots\right)\left(a_{0}+\frac{a_{j} x_{j}}{|x|^{2}}\right) \\
& +\frac{a_{i}}{|x|^{m+2}}-\frac{2 x_{i}}{|x|^{m+4}} a_{j} x_{j}+O\left(|x|^{-m-3}\right) \\
= & -\frac{m}{|x|^{m+2}} a_{0} x_{i}+O\left(|x|^{-m-3}\right) .
\end{aligned}
$$


In these new coordinates we will prove that $v$ is rotationally symmetric about the origin and that $v_{r}<0$ for $r>0$. Note that the equation is rotationally invariant. For any unit vector $\gamma$ we will prove symmetry of $v$ in the plane $\gamma \cdot x=0$ and $\gamma \cdot \operatorname{grad} v<0$ if $\gamma \cdot x>0$. Performing a rotation we may suppose $\gamma=(1,0, \ldots, 0)$. Observe that from $\left(4.3^{\prime}\right)$ we find that for suitable constants $C_{0}, R_{1}$,

$$
v_{1}<0 \quad \text { for } \quad x_{1} \geqq \frac{C_{0}}{|x|} \text { and }|x| \geqq R_{1} \text {. }
$$

As a consequence of (4.3) and (4.4) we first derive

Lemma 4.1. For any $\lambda>0, \exists R=R(\lambda)$ depending only on $\min (1, \lambda)$ (as well as on $v$ ) such that for $x=\left(x_{1}, x^{\prime}\right), y=\left(y_{1}, x^{\prime}\right)$ satisfying

$$
x_{1}<y_{1}, \quad x_{1}+y_{1} \geqq 2 \lambda, \quad|x| \geqq R,
$$

we have

$$
v(x)>v(y) .
$$

Proof. We shall show that if we have a pair of points $x=\left(x_{1}, x^{\prime}\right), y=\left(y_{1}, y^{\prime}\right)$ with $|x| \geqq R_{1} ; y_{1}>x_{1}, y_{1}+x_{1} \geqq 2 \lambda$, and for which the inequality opposite to (4.5) holds, i.e.,

$$
v(x) \leqq v(y),
$$

then necessarily $|x|,|y|<$ some $R$ depending only on $\min (1, \lambda)$. Note that $|y|>|x|$. The proof just involves a bit of tedious calculation using (4.3), (4.4). We will use $C$, $C_{1}$ etc. to denote various constants independent of $\lambda, x$ and $y$. From (4.3) we have (using summation convention)

$$
a_{0}\left(\frac{1}{|x|^{m}}-\frac{1}{|y|^{m}}\right) \leqq a_{i j}\left(\frac{y_{i} y_{j}}{|y|^{m+4}}-\frac{x_{i} x_{j}}{|x|^{m+4}}\right)+C_{1}\left(|x|^{-m-3}+|y|^{-m-3}\right) \leqq C|x|^{-m-2} \text { (4.6) }
$$

Observe that for $p \geqq 1$, since $|y|>|x|$,

$$
\frac{1}{|x|^{p}}-\frac{1}{|y|^{p}} \geqq \frac{1}{|x|^{p-1}}\left(\frac{1}{|x|}-\frac{1}{|y|}\right) \text {. }
$$

As a first consequence of (4.6) we find then

$$
\frac{1}{|x|^{m-1}}\left(\frac{1}{|x|}-\frac{1}{|y|}\right) \leqq \frac{1}{|x|^{m}}-\frac{1}{|y|^{m}} \leqq \frac{C}{|x|^{m+2}}
$$

so that

$$
|y|-|x| \leqq C \frac{|y|}{|x|^{2}} \leqq C\left(\frac{|y|-|x|}{|x|^{2}}+\frac{1}{|x|}\right) .
$$

Hence if $C|x|^{-2} \leqq \frac{1}{2}$ we find

$$
|y|-|x| \leqq \frac{2 C}{|x|} \text { and }|y| \leqq 2|x| .
$$


Inequality (4.8) has been proved assuming $|x|^{2} \geqq 2 C$. We may assume this from now on; for if $|x|^{2} \leqq 2 C$, then since $v(y) \rightarrow 0$ as $|y| \rightarrow \infty$, it follows from (4.5') that $|y| \leqq R$ for some $R$ independent of $\lambda$.

We will now improve the estimate (4.8) by using it in (4.6). Returning to (4.6) we have

$$
\begin{aligned}
a_{0}\left(\frac{1}{|x|^{m}}-\frac{1}{|y|^{m}}\right) \leqq & a_{11}\left(\frac{y_{1}^{2}}{|y|^{m+4}}-\frac{x_{1}^{2}}{|x|^{m+4}}\right) \\
& +2 \sum_{j>1} a_{1 j} x_{j}\left(\frac{y_{1}}{|y|^{m+4}}-\frac{x_{1}}{|x|^{m+4}}\right) \\
& +\sum_{j . k>1} a_{j k} x_{j} x_{k}\left(\frac{1}{|y|^{m+4}}-\frac{1}{|x|^{m+4}}\right) \\
& +C_{1}\left(|x|^{-m-3}+|y|^{-m-3}\right) .
\end{aligned}
$$

As before we may infer that

$$
\begin{aligned}
\frac{1}{|x|^{m-1}}\left(\frac{1}{|x|}-\frac{1}{|y|}\right) \leqq & C \frac{y_{1}^{2}-x_{1}^{2}}{|y|^{m+4}}+C|x|^{2}\left(\frac{1}{|x|^{m+4}}-\frac{1}{|y|^{m+4}}\right) \\
& +C \frac{y_{1}-x_{1}}{|x|^{m+3}}+\frac{C}{|x|^{m+3}} \\
\leqq & C \frac{y_{1}^{2}-x_{1}^{2}}{|x|^{m+4}}+\frac{C}{|x|^{m+1}}\left(\frac{1}{|x|}-\frac{1}{|y|}\right) \\
& +C \frac{y_{1}-x_{1}}{|x|^{m+3}}+\frac{C}{|x|^{m+3}}
\end{aligned}
$$

Using (4.8) we find easily that

$$
|y|-|x| \leqq C \frac{|y|^{2}-|x|^{2}}{|x|^{3}}+C \frac{|y|-|x|}{|x|^{2}}+C \frac{y_{1}-x_{1}}{|x|^{2}}+\frac{C}{|x|^{2}}
$$

Multiplying by $|y|+|x|$ and recalling that

$$
|y|^{2}-|x|^{2}=y_{1}^{2}-x_{1}^{2} \geqq 2 \lambda\left(y_{1}-x_{1}\right)
$$

we find

$$
y_{1}^{2}-x_{1}^{2} \leqq \frac{C}{|x|^{2}}\left(y_{1}^{2}-x_{1}^{2}\right)+\frac{C}{|x|}\left(y_{1}-x_{1}\right)+\frac{C}{|x|} .
$$

Once again, if $|x|^{2} \geqq 2 C$ - as we may assume - it follows that

$$
y_{1}^{2}-x_{1}^{2} \leqq \frac{C}{|x|}\left(y_{1}-x_{1}\right)+\frac{C}{|x|} .
$$

Hence, by (4.9),

$$
\left(2 \lambda-\frac{C}{|x|}\right)\left(y_{1}-x_{1}\right) \leqq \frac{C}{|x|} \text {. }
$$


Thus if $2 \lambda-C|x|^{-1} \geqq \lambda$, i.e.,

$$
|x| \geqq \frac{C}{\lambda}
$$

we see that

$$
y_{1}-x_{1} \leqq \frac{C}{\lambda|x|} .
$$

Consequently, since $y_{1} \geqq 2 \lambda-x_{1}$, we find

$$
2 \lambda-2 x_{1} \leqq \frac{C}{\lambda|x|}
$$

or

$$
x_{1} \geqq \lambda-\frac{C}{2 \lambda|x|} .
$$

But (see (4.4) for $C_{0}$ )

$$
\lambda-\frac{C}{2 \lambda|x|} \geqq \frac{C_{0}}{|x|}
$$

provided.

$$
|x| \geqq \frac{C}{2 \lambda^{2}}+\frac{C_{0}}{\lambda} .
$$

Thus if (4.11) and (4.10) hold we conclude that

$$
x_{1} \geqq \frac{C_{0}}{|x|} .
$$

But then (4.4) implies that $v$ is strictly decreasing on the straight segment going from $x$ to $y$-contradicting $\left(4.5^{\prime}\right)$. Thus either $(4.10)$ or $(4.11)$ cannot hold and the lemma is proved.

As in Sect.2, for any $\lambda>0$ and for any $x=\left(x_{1}, x^{\prime}\right)$, we denote by $x^{\lambda}$ its reflection in the plane $x_{1}=\lambda$, i.e., $x^{\lambda}=\left(2 \lambda-x_{1}, x^{\prime}\right)$.

Lemma 4.2. There exists $\lambda_{0} \geqq 1$ such that $\forall \lambda \geqq \lambda_{0}$,

$$
v(x)>v\left(x^{\lambda}\right) \text { if } \quad x_{1}<\lambda .
$$

Proof. Set $R_{1}=\max \left\{1, R(1)\right.$ of Lemma 4.1\}. By Lemma 4.1, if $|x|>R_{1}, \lambda \geqq 1$ and $x_{1}<\lambda$ we have

$$
v(x)>v\left(x^{\hat{\lambda}}\right)
$$

But

$$
v(x) \geqq c_{0}>0 \quad \text { for } \quad|x| \leqq R_{1} .
$$


Furthermore for $1<R_{2}$ sufficiently large we have

$$
v(y)<c_{0} \quad \text { for } \quad|y| \geqq R_{2} .
$$

Thus (4.12) holds if $\lambda \geqq R_{2}$ and $|x| \leqq R_{1}$. Combining this with (4.12') we obtain (4.12) with $\lambda_{0}=R_{2}$. Q.e.d.

Lemma 4.2 asserts the desired reflection property (4.12) for planes $T_{\lambda}: x_{1}=\lambda$ with $\lambda$ sufficiently large. Now we may begin our procedure of moving the plane $T_{\lambda}$ by decreasing $\lambda$. First we have the analogue of Lemma 2.2.

Lemma 4.3. Assume that for some $\lambda>0$

$$
v(x) \geqq v\left(x^{\lambda}\right), \quad v(x) \neq v\left(x^{\lambda}\right) \text { for } \quad x_{1}<\lambda,
$$

Then $v(x)>v\left(x^{\lambda}\right)$ if $x_{1}<\lambda$, and

$$
v_{1}(x)<0 \text { on } T_{\lambda} \text {. }
$$

Proof. The function $w(x)=v\left(x^{\lambda}\right)$ is also a solution of (1.8) in $x_{1}<\lambda$ and $w \leqq v$ there. Thus the function

$$
z(x)=w(x)-v(x) \leqq 0, \quad z \neq 0
$$

satisfies an elliptic equation of the form

$$
L w=0 \quad \text { in } \quad x_{1} \leqq \lambda
$$

with $L$ as in (1.10), and it achieves its maximum, namely zero, at every point on $T_{j}$. By the maximum principle and Lemma $\mathrm{H}, z<0$ and

$$
0<z_{1}=-2 v_{1} \text { on } T_{\lambda} .
$$

The lemma is proved.

Lemma 4.4. The set of positive $\lambda$ for which (4.12) holds is open.

Proof. Suppose (4.12) holds for $\lambda=\bar{\lambda}>0$. Set $\bar{R}=R(\bar{\lambda} / 2)$ of Lemma 4.1; then (4.12) holds for $\lambda \geqq \bar{\lambda} / 2$ provided $|x|>\bar{R}$. We have only to consider $|x| \leqq \bar{R}$. If (4.12) did not hold for all $\lambda$ in some neighbourhood of $\bar{\lambda}$ there would be a sequence $\left\{x^{j}\right\}$, $j=1,2, \ldots$ in $\left|x^{j}\right| \leqq R$ and a sequence $\lambda^{j} \rightarrow \bar{\lambda}, \lambda^{j} \geqq \bar{\lambda} / 2$, with $x_{1}^{j}<\lambda^{j}$ and

$$
v\left(x^{j}\right) \leqq v\left(x^{j \bar{\lambda}^{j}}\right) .
$$

Then a subsequence, which we still call $x^{j}$, converges to some $x$ in $|x| \leqq R$ and

$$
v(x) \leqq v\left(x^{\bar{\lambda}}\right) .
$$

In view of (4.12) it follows that $x_{1}=\bar{\lambda}$. But then from (4.14) we must have

$$
v_{1}(x) \geqq 0
$$

- contradicting Lemma 4.3. Q.e.d.

From Lemmas 4.2, 4.4 and 4.3 we may conclude that (4.12) and (4.13) hold for all $\lambda$ in some maximal open interval $0 \leqq \lambda_{1}<\lambda<\infty$. In particular we also have

$$
v_{1}(x)<0 \text { for } x_{1}>i_{1} .
$$


In addition, by continuity

$$
v(x) \geqq v\left(x^{\lambda_{1}}\right) \quad \text { if } \quad x_{1}<\lambda_{1} .
$$

Corresponding to the direction $\gamma$ which we took to be $(1,0, \ldots, 0)$, we have found a maximal open interval

$$
0 \leqq \lambda_{1}(v)<\lambda<\infty
$$

such that the reflection property (4.12) holds for all $\lambda$ in the interval.

Suppose for some vector $\gamma$, which we may take as $(1,0, \ldots, 0), \lambda_{1}(\gamma)>0$. By Lemma 4.3 we either have

$$
v(x) \equiv v\left(x^{\lambda_{1}}\right), \quad \text { for } \quad x_{1}<\lambda_{1},
$$

or else property (4.12) holds for $\lambda_{1}$. The former cannot occur, by Lemma 4.1, while the latter cannot, by Lemma 4.4 and the definition of $\lambda_{1}$. Hence for all unit vectors $\gamma, \lambda_{1}(\gamma)=0$. It follows from (4.16) that $v$ is symmetric about each plane $\gamma \cdot x=0$. Hence it is radially symmetric about the origin, as was to be proved.

The remainder of the theorem follows from (4.15).

Remark. Theorem 4 yields rotational symmetry of our solution. If we wish to prove symmetry in only one direction, say with respect to $x_{1}$ then it is clear that the argument extends to more general equations than (1.8). For example we may consider elliptic equations of the form (here $\alpha, \beta$ range from 2 to $n$ )

$$
F\left(x_{2}, \ldots, x_{n}, v, v_{1}^{2}, v_{2}, \ldots, v_{n}, v_{11}, v_{\alpha \beta}\right)=0
$$

Theorem 4'. Let $v>0$ be a $C^{2}$ solution of (4.17) in $\mathbb{R}^{n}$ with $F, F_{v}, F_{v_{1}^{2}}, \ldots, F_{v_{\alpha \beta}} \in C$ and $v$ satisfying (1.9). Then $v$ is symmetric with respect to the plane $x_{1}=-\frac{a_{1}}{m a_{0}}$ and $v_{1}<0$ for $x_{1}>-\frac{a_{1}}{m a_{0}}$.

\section{Parabolic Equations}

For parabolic equations the maximum principle as well as its Corollary 1 , and the Hopf boundary lemma (see [7], Chapter 3, §3) hold. Therefore all our results admit extensions to such equations with essentially the same proofs. We shall content ourselves with the statement of several results for functions $u(t, x)$ defined in a cylinder $\Omega=(0<t<T) \times G, G$ is a bounded domain in $\mathbb{R}^{n}$ with $\partial G$ smooth. By a (maximal) cap of $\Omega$ we always mean $[0<t<T) \times[$ a (maximal) cap of $G]$.

Condition I. With $G=R^{\prime}<|x|<R$, let $u$ be a positive solution of

$$
-u_{t}+\Delta u+f(t, r, u)=0, \quad u=0 \quad \text { on }|x|=R
$$

with $u_{t}, u_{j}, u_{k \ell}$ belonging to $C$ in the closure. Here $f$ and $f_{u}$ are continuous in $\bar{G} \times R^{+}$. Assume that $f$ is decreasing in $r$ and that $u(0, x)$, is rotationally symmetric and decreasing in $r$. 
Theorem 5.1. Assume Condition I. Then

$$
u_{r}<0 \quad \text { for } \quad \frac{R+R^{\prime}}{2} \leqq r<R
$$

If, in addition, $u$ is a solution in $|x|<R$, then $\forall t, 0<t<T, u$ is radially symmetric and $u_{r}<0$ for $0<r<R$.

The next result extends Theorem $2.1^{\prime}$ in which we consider a maximal cap $\Omega \cap\left\{x_{1}>\lambda_{1}\right\}$.

Condition II. Consider a positive solution in $G$ of

$$
-u_{t}+F\left(t, x, u, u_{i}, u_{j k}\right)=0, \quad u=0 \quad \text { on } \quad \partial G
$$

with $F$ as in Section 2.4 satisfying in particular condition (c), and assume $u(0, x)$ satisfies condition (2.2).

Theorem 5.2. Assume Condition II. Then $\forall t$ in $0<t<T, u(t, x)$ satisfies condition (2.2). Furthermore if $u_{1}=0$ at some point $\left(x, t_{0}\right)$ in $\Omega \cap\left\{x_{1}=\lambda_{1}\right\}$ then for $0<t \leqq t_{0}, u$ is symmetric about the plane $x_{1}=\lambda_{1}$ and (2.3) holds.

The results in Sect. 3 also admit extensions to parabolic equations.

\section{Appendix. Lemma $S$ and Related Results}

One may ask what happens if condition (1.12) in Lemma $S$ is dropped. The following example shows that it is essential. In $R^{2}$, using polar coordinates, the function

$$
w=-r^{\pi / \theta_{0}} \sin \frac{\pi \theta}{\theta_{0}}
$$

is negative in the angle $\Omega: 0<\theta<\theta_{0}<\pi / 2$, vanishes on the boundary and satisfies $\Delta w=0$. But it does not satisfy (1.13); here $a_{i j} \varrho_{i} \sigma_{j}<0$ at the origin.

Since it may be of interest we will also prove a rather primitive extension of Lemma $S$ to the case where condition (1.12) does not hold. The preceding example is then seen to be typical.

Lemma A.1. Let $\Omega$ be as in Lemma $S$. Let $w \in C^{2}(\Omega) \cap C(\bar{\Omega})$, with $w<0$ in $\Omega, w(0)=0$, be a solution of the elliptic differential inequality (1.10) in $\Omega$. Assume that the leading coefficients $a_{i j} \in C(\bar{\Omega})$, and satisfy (1.11), and that the others are bounded. In place of (1.12) assume that

$$
\sum a_{i j} \varrho_{i} \sigma_{j}=\mu \sqrt{\sum a_{i j} \varrho_{i} \varrho_{j}} \sqrt{a_{i j} \sigma_{i} \sigma_{j}} \text { at } 0
$$

for some constant $\mu$; clearly $-1<\mu<1$. Set $\theta_{0}=\arccos (-\mu)$.

Suppose

$$
p>\frac{\pi}{\theta_{0}}
$$


Let $\mathscr{C}$ be a closed cone with vertex at the origin such that for some $\varepsilon>0$, $\mathscr{C} \cap\{0<|x|<\varepsilon\}$ lies in $\Omega$. Then there is a positive constant $\delta$ and a neighbourhood in $\mathscr{C}$ of 0 in which

$$
w+\delta|x|^{p} \leqq 0 .
$$

In particular if $\mu>0$ we may take $p<2$ and it follows that if $w \in C^{1}(\bar{\Omega})$ and $\operatorname{grad} w(0)=0$, then on any ray from 0 entering $\Omega$ transversally to the surface, the second derivative of $w$ cannot be bounded near the origin. On the other hand, if $\mu<0$ and $w \in C^{r}$ in $\bar{\Omega}^{1}$ near 0 , for $r>\pi / \theta_{0}$, then at least one of the derivatives

$$
\left(\frac{\partial}{\partial s}\right)^{j} w, \quad j=1, \ldots,\left[\frac{\pi}{\theta_{0}}\right]
$$

is negative at 0 .

The following may also prove useful.

Lemma A.2. Let $\Omega$ be a component of a cone: $\phi=b^{i j} x_{i} x_{j}>0$. Assume $w \in C^{2}(\Omega) \cap C(\bar{\Omega})$ near the origin, that $w<0$ in $\bar{\Omega}$ except at the origin, $w(0)=0$, and that $w$ satisfies (1.10) in $\Omega$. Let $p$ be such that the quadratic form

$$
Q \equiv(p-1) a_{i j}(0) \phi_{i} \phi_{j}+\phi a_{i j}(0) \phi_{i j}>0, \quad x \neq 0, \quad x \in \bar{\Omega} .
$$

Then there is a $\delta>0$ and a neighbourhood of $O$ in $\Omega$ in which

$$
w+\delta \phi^{p}<0 .
$$

Both lemmas will be proved at the end of the Appendix.

Our proof of Lemma $S$ is an extension of Serrin's; it makes use of suitable comparison functions.

Proof of Lemma $S$. We first remark that it suffices to prove it in case $c(x) \equiv 0$. The general case then follows by the same argument we presented for Lemma $H$. The case of strict inequality in (1.12) follows from Lemma A.1. So we just consider the case of equality.

We may suppose that $w<0$ in $\bar{\Omega} \backslash 0$ for we may simply replace the hypersurfaces by spheres tangent to them at 0 and lying, otherwise entirely in $\Omega$.

In the closure $\bar{\Omega}_{\varepsilon}$ of $\Omega_{\varepsilon}=\Omega \cap\{|x|<\varepsilon\}$ we will construct a $C^{2}$ function $z$ with the properties :

$$
\begin{aligned}
& \left\{\begin{array}{c}
z \leqq 0 \quad \text { on } \quad \varrho=0 \quad \text { and on } \quad \sigma=0, \\
z(0)=z_{s}(0)=0, \quad z_{s s}(0)>0,
\end{array}\right. \\
& L z \geqq 0 .
\end{aligned}
$$

Using such a function the proof is easily carried out. Set

$v=w+t z$

1 If $r$ is not an integer this means: $w \varepsilon C^{[r]}$, and the derivatives of $w$ of order [r] satisfy a Hölder condition with exponent $r-[r]$ 
with $t>0$ so small that $w+t z \leqq 0$ on the part of the boundary of $\bar{\Omega}_{\varepsilon}$ where $|x|=\varepsilon$; recall that $w<0$ on that compact set. On the other parts of the boundary of $\bar{\Omega}_{\varepsilon}$ we have $v \leqq w$. Thus $v \leqq 0$ on the whole boundary of $\bar{\Omega}_{\varepsilon}$. In $\Omega_{\varepsilon}$ we have

$$
L v \geqq 0 \text {. }
$$

By the maximum principle $v$ achieves its maximum on the boundary - in particular at the point 0 . Thus $v_{s}(0) \leqq 0$ and if $v_{s}(0)=0$ then $v_{s s}(0) \leqq 0$. Now $v_{s}(0)$ $=w_{s}(0)$ and $v_{s s}(0)=w_{s s}(0)+t z_{s s}(0)>w_{s s}(0)$, and (1.13) follows.

After a $C^{2}$ change of variables we may suppose that the new surfaces are given by $x_{1}=0$ and $x_{n}=0$ and $\Omega$ is in $\left\{x_{1}<0\right\} \cap\left\{x_{n}<0\right\}$. Condition (1.12) now takes the form

$$
a_{1 n}(0)=0 \text {. }
$$

We proceed in several steps.

(i) First we make a linear change of variable so that in the new variables we also have

$$
a_{1 \alpha}(0)=a_{x n}(0)=0 \quad \text { for } 1<\alpha<n .
$$

Set

$$
\begin{aligned}
& y_{1}=x_{1}, \quad y_{n}=x_{n} \\
& y_{\alpha}=x_{\alpha}+c_{\alpha} x_{1}+d_{\alpha} x_{n}
\end{aligned}
$$

with constants $c_{\alpha}, d_{\alpha}$ to be chosen. The boundaries $x_{1}=0, x_{n}=0$ become $y_{1}=0$, $y_{n}=0$. Let us compute, using summation convention $(\alpha, \beta, \gamma$ are summed from 2 to $n-1)$ :

$$
\begin{aligned}
a_{i j} \partial_{x_{i}} \partial_{x_{j}}= & a_{11}\left(\partial_{y_{1}}+c_{\alpha} \partial_{y_{\alpha}}\right)\left(\partial_{y_{1}}+c_{\beta} \partial_{y_{\beta}}\right) \\
& +2 a_{1 \gamma}\left(\partial_{y_{1}}+c_{\alpha} \partial_{\gamma_{\alpha}}\right) \partial_{y_{\gamma}} \\
& +2 a_{1 n}\left(\partial_{y_{1}}+c_{\alpha} \partial_{\gamma_{\alpha}}\right)\left(\partial_{y_{n}}+d_{\alpha} \partial_{y_{\alpha}}\right) \\
& +a_{\alpha \beta} \partial_{v_{\alpha}} \partial_{y_{\beta}}+2 a_{\gamma_{n}}\left(\partial_{y_{n}}+d_{\alpha} \partial_{y_{\alpha}}\right) \partial_{y_{\gamma}} \\
& +a_{n n}\left(\partial_{y_{n}}+d_{\alpha} \partial_{y_{\alpha}}\right)\left(\partial_{y_{n}}+d_{\beta} \partial_{v_{\beta}}\right) \\
\equiv & b_{i j} \partial_{y_{i}} \partial_{y_{j}} .
\end{aligned}
$$

Thus

$$
\begin{aligned}
& b_{1 n}(0)=a_{1 n}(0)=0 \\
& b_{1 \alpha}(0)=a_{11}(0) c_{\alpha}+a_{1 \alpha}(0) \quad \alpha=2, \ldots, n . \\
& b_{\alpha n}(0)=a_{n n}(0) d_{\alpha}+a_{\alpha n}(0)
\end{aligned}
$$

We now require that these be zero - determining the $c_{\alpha}$ and $d_{\alpha}$.

For convenience we will continue to call the new variables $x_{j}$ and the coefficients of $L, a_{i j}$ etc. They now satisfy (A.6). In the new coordinates condition $\left(1.12^{\prime}\right)$ means that

$$
\partial_{x_{\alpha}} a_{1 n}(0)=0, \quad 1<\alpha<n .
$$


(ii) We will now replace $\Omega$ by a slightly smaller region. With $k$ to be chosen suitably large, set

$$
\begin{aligned}
& \phi=x_{1}+k \sum_{2}^{n-1} x_{\alpha}^{2}, \\
& \psi=x_{n}+k \sum x_{\alpha}^{2} .
\end{aligned}
$$

Consider the smaller region $G=\Omega \cap\{\phi<0\} \cap\{\psi<0\}$. In $G$ near $O$ we will use the comparison function

$$
z=g h
$$

with

$$
g=e^{-\alpha \phi}-1, \quad h=e^{-\alpha \psi}-1
$$

and $\alpha$ suitably large. Clearly

$$
\begin{aligned}
z & >0, \\
z & =0 \quad \text { on } \phi=0 \text { and on } \psi=0, \\
z_{s}(0) & =0, \quad z_{s s}(0)>0 .
\end{aligned}
$$

We will choose $k, \alpha$ and an $\varepsilon$ neighbourhood $G_{\varepsilon}$ of $O$ in $G$ so that $z$ satisfies

(A.3) in $G_{\varepsilon}$.

(iii) Computing, we have

$$
L z=g L h+h L g+2 a_{i j} g_{i} h_{j} .
$$

Here $g_{i}=g_{x_{2}}$ etc. Now (here $\beta, \gamma$ are summed from 2 to $n-1$ ) for $\alpha$ large:

$$
\begin{aligned}
e^{\alpha \phi} \frac{L g}{\alpha^{2}} & =a_{i j} \phi_{i} \phi_{j}-\frac{1}{\alpha}\left(a_{i j} \phi_{i j}+b_{i} \phi_{i}\right) \geqq c_{0}>0 . \\
I & =\alpha^{-2} e^{\alpha(\phi+\psi)} a_{i j} g_{i} h_{j}=a_{i j} \phi_{i} \psi_{j} \\
& =a_{1 n}+2 k a_{1 \gamma} x_{\gamma}+4 k^{2} a_{\gamma \beta} x_{\gamma} x_{\beta}+2 k a_{n \gamma} x_{\gamma} .
\end{aligned}
$$

We first choose $k$ so that $I$ is nonnegative on $\{\phi=0\} \cap\{\psi=0\}$, i.e., for points of the form

$$
\left(x_{1}, \ldots, x_{n}\right)=\left(-k \sum x_{x}^{2}, x_{2}, \ldots, x_{n-1},-k \sum x_{x}^{2}\right) .
$$

At such points, because of (A.7) we have

$$
\left|a_{1 n}\right| \leqq C\left(\left|x_{1}\right|+\left|x_{n}\right|+\sum x_{\alpha}^{2}\right)
$$

with some constant $C$. $C$ will denote various constants independent of $k$ and $\alpha$. Therefore, supposing $k \geqq 1$,

$$
\left|a_{1 n}\right| \leqq C k \sum x_{\alpha}^{2} \text {. }
$$

In addition, from (A.6) we see that

$$
\begin{aligned}
\left|a_{1 \alpha}\right|,\left|a_{x n}\right| & \leqq C|x| \leqq C\left(\sum\left|x_{\beta}\right|+k \sum x_{\beta}^{2}\right) \\
& \leqq C \sum\left|x_{\beta}\right|
\end{aligned}
$$


provided we require

$$
k \sum\left|x_{\beta}\right| \leqq 1 .
$$

Inserting these in (A.10) we find

$$
\begin{aligned}
I & \geqq 4 k^{2} a_{\alpha \beta} x_{\alpha} x_{\beta}-C k \sum x_{\alpha}^{2}-C k \sum x_{\alpha}^{2} \\
& \geqq\left(c_{0} k^{2}-C k\right) \sum x_{\alpha}^{2} \text { for some } \quad c_{0}>0
\end{aligned}
$$

by uniform ellipticity. We now fix $k \geqq C / c_{0}$ and infer

$$
I \geqq 0 \quad \text { on } \quad\{\phi=0\} \cap\{\psi=0\} \cap\left\{\sum\left|x_{\alpha}\right|<k^{-1}\right\} .
$$

It follows that, with $k$ so fixed

$$
I \geqq C(\phi+\psi) \quad \text { in } \quad G \cap\{|x|<\delta\}
$$

for some small $\delta$ independent of $\alpha$.

(iv) Next, for $\alpha$ large, we have

$$
\begin{aligned}
\frac{1}{\alpha^{2}} e^{\alpha \phi} L g & =a_{i j} \phi_{i} \phi_{j}-\frac{1}{\alpha}\left(a_{i j} \phi_{i j}+b_{i} \phi_{i}\right) \\
& \geqq c_{0}>0
\end{aligned}
$$

for some positive constant $c_{0}$. A similar estimate holds for $L h$. Inserting these in (A.9) and using (A.10) and (A.11) we find

$$
\frac{1}{\alpha^{2}} e^{\alpha(\phi+\psi)} L z \geqq c_{0}\left(1-e^{\alpha \phi}+1-e^{\alpha \psi}\right)+2 C(\phi+\psi)
$$

in $G \cap\{|x|<\delta\}$.

By the theorem of the mean, for $\phi \leqq 0$,

$1-e^{\alpha \phi} \geqq-\alpha e^{\alpha \phi} \phi$.

Let us now restrict ourselves to the region

$$
-\frac{1}{\alpha}<\phi, \psi \leqq 0, \quad \alpha \text { large }
$$

Then

$$
\begin{aligned}
& 1-e^{\alpha \phi} \geqq-\frac{\alpha}{e} \phi \\
& 1-e^{\alpha \psi} \geqq-\frac{\alpha}{e} \psi .
\end{aligned}
$$

Inserting these in (A.12) we find

$$
\frac{1}{\alpha^{2}} e^{\alpha(\phi+\psi)} L z \geqq\left(\frac{c_{0} \alpha}{e}-2 C\right)(|\phi|+|\psi|) \geqq 0
$$


for $\alpha$ large - in the region

$$
\left\{-\frac{1}{\alpha}<\phi, \psi \leqq 0\right\} \cap\{|x|<\delta\}=G_{\alpha, \delta}
$$

With $\alpha$ so fixed, in the region $G_{x, \delta}$, the function $z$ has all the desired properties. The proof is complete.

We turn now to Lemmas A.1 and A.2. As before it suffices to consider the case that $c(x) \equiv 0$.

First, the

Proof of Lemma A.2. We need only show that near $O$ in $\Omega$ the function

$$
z=\phi^{p}
$$

satisfies $L z \geqq 0$. Then the proof proceeds as before.

A computation yields [see $\left(\right.$ A. $\left.\left.1^{\prime}\right)\right]$

$$
\begin{aligned}
\frac{1}{p} \phi^{2-p} L z & =(p-1) a_{i j} \phi_{i} \phi_{j}+\phi L \phi \\
& =Q+O\left(|x|^{3}\right) \\
& \geqq 0 \quad \text { in } \bar{\Omega} \text { near } O
\end{aligned}
$$

by (A.1'). Q.e.d.

Proof of Lemma A.1. As before, we may suppose $w<0$ in $\bar{\Omega} \backslash 0$. After a smooth change of variable we may suppose that the boundary hypersurfaces are hyperplanes $x_{1}=0$ and $x_{n}=0$. By a further linear transformation of the variables $x_{1}, x_{n}$ we may suppose that at 0 ,

$$
a_{11}=a_{n n}=1, \quad a_{1 n}=0,
$$

and that $\Omega$ is the wedge:

$$
x_{n}>0, \quad x_{1}>x_{n} \cot \theta_{0}
$$

where $\mu=-\cos \theta_{0}$. After these changes of variables the cone $\mathscr{C}$ has become a deformed cone, but it suffices to prove the result in the new $\Omega$ for any closed cone in $\Omega$ as before - which we still denote as $\mathscr{C}$.

Near the origin in $\bar{\Omega}$ we shall construct a function $z \in C^{2}(\Omega) \cap C(\bar{\Omega})$ satisfying

$$
\begin{aligned}
z & =0 \quad \text { on } \quad \partial \Omega \\
z(x) & \geqq c_{0}|x|^{p} \text { in } \mathscr{C}, \text { with } c_{0}>0 \\
L z & \geqq 0 \text { in } \Omega .
\end{aligned}
$$

As in the proof of Lemma $S$ we find that for some small $t>0, w+t z \leqq 0$ in $\Omega_{\varepsilon}$, and the desired conclusion follows.

Introduce the complex variable

$$
\zeta=x_{1}+i x_{n} .
$$


The wedge $\Omega$ is then given by $0<\arg \zeta<\theta_{0}$ and on $\Omega$ the function

$$
v=\operatorname{Im}\left(\zeta^{\pi / \theta_{0}}\right)
$$

is harmonic in the variables $\left(x_{1}, x_{n}\right)$ and vanishes on $\partial \Omega$. For $k=p \theta_{0} / \pi>1$ set

$$
z=v^{k} \text {. }
$$

Then $z$ satisfies (A.13) and (A.14), and we will prove that $z$ satisfies (A.15) - the proof of the lemma will then be complete.

Near the origin in $\Omega$ we have, for $i, j=1, n$,

$$
\begin{aligned}
z_{x_{\imath}} & =k v^{k-1} v_{x_{\imath}}=O\left(|\zeta|^{p-1}\right) \\
z_{x_{\imath} x_{J}} & =k v^{k-1} v_{x_{\imath} x_{J}}+k(k-1) v^{k-2} v_{x_{\imath}} v_{x_{J}}=O\left(|\zeta|^{p-2}\right) .
\end{aligned}
$$

Thus

$$
\begin{aligned}
z_{x_{1} x_{1}}+z_{x_{n} x_{n}} & =k(k-1) v^{k-2}\left(v_{x_{1}}^{2}+v_{x_{n}}^{2}\right) \\
& \geqq c_{1}|\zeta|^{p-2} \quad \text { for some } \quad c_{1}>0 .
\end{aligned}
$$

Since $L z=z_{x_{1} x_{1}}+z_{x_{n} x_{n}}$ at 0 , and the leading coefficients of $L$ are continuous, with the others bounded, it follows that

$$
L z \geqq 0
$$

in $\Omega$ near the origin.

\section{References}

1. Corrigan, F., Fairlie, D.: Scalar field theory and exact solutions in a classical $S U(2)$ gauge theory. Phys. Lett. 67 B, 69 (1977)

2. Courant, R., Hilbert, D.: Methods of mathematical physics. Interscience-Wiley, Vol. II (1962)

3. Hopf, H.: Lectures on differential geometry in the large. Stanford University, 1956

4. Jackiw, R., Rebbi, C.: Conformal properties of pseudoparticle configurations. Phys. Rev. D 16, 1052 (1976)

5. Loewner, C., Nirenberg, L. : Partial differential equations invariant under conformal and projective transformations. In: Contributions to Analysis, pp. 245-272. Academic Press 1974

6. Obata, M.: The conjectures on conformal transformations of Riemannian manifolds. J. Diff. Geom. 6, 247--258 (1971)

7. Protter, M., Weinberger, H.: Maximum principles in differential equations. Prentice-Hall 1967

8. Serrin, J.: A symmetry problem in potential theory. Arch. Ration. Mech. 43, 304-318 (1971)

9. Wilczek, F.: Geometry and interactions of instantons. In: Quark confinement and field theory. Stump, D., Weingarten, D. (eds.). New York: Wiley 1977

Communicated by A. Jaffe

Received March 9, 1979 
